\title{
Weak Lensing Effects on the Galaxy Three-Point Correlation Function
}

\author{
Fabian Schmidt, ${ }^{1,2}$ Alberto Vallinotto, ${ }^{3}$ Emiliano Sefusatti, ${ }^{4}$ and Scott Dodelson ${ }^{1,2,4}$ \\ ${ }^{1}$ Department of Astronomy $\&$ Astrophysics, The University of Chicago, Chicago, IL 60637-1433 \\ ${ }^{2}$ Kavli Institute for Cosmological Physics, Chicago, IL 60637-1433 \\ ${ }^{3}$ Institute d'Astrophysique de Paris, CNRS-UMR 7095, \\ Université Paris VI Pierre et Marie Curie, Paris, France \\ ${ }^{4}$ Particle Astrophysics Center, Fermi National Accelerator Laboratory, Batavia, IL 60510-0500
}

(Dated: October 24, 2018)

\begin{abstract}
We study the corrections to the galaxy three-point correlation function (3PCF) induced by weak lensing magnification due to the matter distribution along the line of sight. We consistently derive all the correction terms arising up to second order in perturbation theory and provide analytic expressions as well as order of magnitude estimates for their relative importance. The magnification contributions depend on the geometry of the projected triangle on the sky plane, and scale with different powers of the number count slope and redshift of the galaxy sample considered. We evaluate all terms numerically and show that, depending on the triangle configuration as well as the galaxy sample considered, weak lensing can in general significantly contribute to and alter the three-point correlation function observed through galaxy and quasar catalogs.
\end{abstract}

PACS numbers: 98.80.-k 98.62.Py 95.35.+d

Keywords: cosmology: theory - large-scale structure of the Universe

\section{INTRODUCTION}

The detection and measurement of the three-point and higher order correlation functions in position space and of their Fourier counterparts in the large-scale galaxy distribution date back to the late seventies and early eighthies, see, e.g. [1-4]. At that time such observations served in the first place as a confirmation of the hierarchical model of structure formation. Cosmological perturbation theory, for instance, makes specific predictions for matter higher order correlators which are generated by non-linearities in the equations describing gravitational evolution, even when Gaussian initial conditions are assumed $[5,6]$. An extra source of non-Gaussianity is given by non-linearities in the galaxy bias, i.e., in the relation between the observed galaxy distribution and the underlying dark matter fluctuations [7].

The measurement of the galaxy bispectrum or of the three-point function in redshift surveys can provide a determination of linear galaxy bias independently of the normalization of the dark matter perturbations [8-10], and, in the framework of the halo model, on the parameters controlling the halo occupation distribution [11, 12]. This kind of analysis has been performed for current large-scale galaxy surveys such as the $2 \mathrm{dF}$ Galaxy Redshift Survey (2dFGRS) [13-15], and the Sloan Digital Sky Survey (SDSS), [16, 17]. More recently, it has been shown that the bispectrum can also provide insights into the non-linear nature of galaxy bias which can be used to constrain models of the power spectrum in the range of the acoustic oscillations [18-20]. In general, it has been shown that in surveys such as the SDSS, adding bispectrum information allows for an improvement in the determination of cosmological parameters as compared to using the power spectrum alone [21].

An additional contribution to the galaxy three-point function is expected from primordial non-Gaussianity, i.e. if perturbations at early times are not perfectly Gaussian [22-28]. Current constraints on primordial nonGaussianities derived from CMB observations, [29-33] still allow for a component potentially observable in future large-scale galaxy surveys, [27, 34].

Recent interest in the observation of acoustic features in the galaxy correlation as a means to accurately map the expansion history of the Universe and thereby constrain dark energy parameters [35-38], has motivated several proposals for future large surveys at high redshift, as, for instance, the Hobby-Eberly Dark Energy Experiment (HETDEX) [39], the Wide-Field Multi-Object Spectrograph (WFMOS) [40], the Advanced Dark Energy Physics Telescope (ADEPT) mission, the ESA Euclid mission [41], or the Cosmic Inflation Probe (CIP) [42].

One of the technical and theoretical challenges that these projects will have to face is that the galaxy power spectrum or correlation function of high-redshift objects is affected by weak lensing magnification due to the intervening matter along the line-of-sight [43-47]. When a magnitude limited survey is carried out, weak gravitational lensing acts on what is observed in several ways. The first effect arises because a congruence of photons traveling from a distant galaxy to the observer is focused or defocused by gravitational potentials along the way, thus resulting in magnification or demagnification of the object observed. Weak lensing therefore pushes objects over the threshold magnitude of the survey, in either direction. The resulting catalog does not contain all the objects that are intrinsically brighter than the limiting magnitude, and contains some objects that are intrinsically fainter than this magnitude. The size of this effect depends on the slope of the magnitude distribution of galaxies: the steeper the slope, the stronger the effect of 
weak lensing magnification on the observed number density will be. The second effect is due to the fact that weak lensing also stretches or contracts patches of the sky, thus varying the density of objects observed. Through these two first-order effects, lensing affects the observation of galaxy overdensities. Since these are used as a way of quantifying matter overdensities, weak lensing enters the measurement of any correlation function that is based on a magnitude limited survey. We do not consider the effects of the lensing-induced displacement of sources here (i.e. the "smoothing effect"), as we expect it to be smaller than the magnification contribution.

This work attempts a first step towards quantifying and possibly exploiting the weak lensing contribution to the three point correlation function of pointlike objects like galaxies or quasars.

The intrinsic galaxy three-point function depends on three parameters (e.g., the three sides of a triangle). The lensing effects induce an orientation dependence in the observed 3PCF, specified e.g. by two angles. Hence, the lensing effects depend on five parameters, in addition to the number count slope and the redshift considered. In this paper, we do not attempt to fully explore this vast parameter space. Rather, after presenting the full set of equations, we focus on specific parameter sets chosen to allow for an understanding of the basic features of the lensing effects.

For this work, we use the framework of second order cosmological perturbation theory $[5,6]$. While the validity of this approach is limited to large scales, it allows for a straightforward and consistent analytical treatment of the magnification correction. Moreover, the results are physically intuitive, and, as we will show, all relevant expressions reduce to manageable integrals over a small number of auxiliary functions.

The paper is organized as follows. In Sec. II we present the un-lensed 3PCF, we then derive all the terms arising because of weak lensing and provide order of magnitude estimates for the different correction terms. In Sec. III we present results for four specific triangular configurations and for the reduced three-point function. We also obtain an estimate of the observability of the lensing effect for future surveys. We conclude in Sec. IV. The details of the calculation as well as discussions of approximations used are relegated to the Appendices.

\section{MAGNIFICATION OF THE 3PCF}

\section{A. The un-lensed galaxy 3-point correlation function}

We want to study the effects of lensing magnification on the observable galaxy 3-point correlation function (3PCF) on large scales. A non-vanishing three-point correlation function, even for Gaussian initial conditions, is expected due to non-linearities in the dark matter evolution, as well as due to non-linearities in the galaxy bias, i.e. the relation between the observed galaxy distribution and the underlying dark matter field.

Here we consider the expression for the dark matter $3 \mathrm{PCF}$ predicted by tree-level perturbation theory (PT) [5], for a review see [6]. The dark matter bispectrum, with Gaussian initial conditions, is then given by

$$
B\left(k_{1}, k_{2}, k_{3}\right)=F_{2}\left(\mathbf{k}_{1}, \mathbf{k}_{2}\right) P_{L}\left(k_{1}\right) P_{L}\left(k_{2}\right)+2 \text { perm., }
$$

where $P_{L}(k)$ is the dark matter linear power spectrum and the PT kernel $F_{2}$ is given by

$$
F_{2}\left(\mathbf{k}_{1}, \mathbf{k}_{2}\right)=\frac{5}{7}+\frac{1}{2} \frac{\mathbf{k}_{1} \cdot \mathbf{k}_{2}}{k_{1} k_{2}}+\frac{2}{7}\left(\frac{\mathbf{k}_{1} \cdot \mathbf{k}_{2}}{k_{1} k_{2}}\right)^{2} .
$$

The three-point matter correlation function in position space is defined as

$$
\begin{aligned}
\zeta\left(\mathbf{x}_{1}, \mathbf{x}_{2}, \mathbf{x}_{3}\right) \equiv & \left\langle\delta\left(\mathbf{x}_{1}\right) \delta\left(\mathbf{x}_{2}\right) \delta\left(\mathbf{x}_{3}\right)\right\rangle \\
= & \int d^{3} k_{1} e^{-i \mathbf{k}_{1} \cdot \mathbf{x}_{1}} \int d^{3} k_{2} e^{-i \mathbf{k}_{2} \cdot \mathbf{x}_{2}} \\
& \times \int d^{3} k_{3} e^{-i \mathbf{k}_{3} \cdot \mathbf{x}_{3}} \delta_{D}\left(\mathbf{k}_{123}\right) B\left(k_{1}, k_{2}, k_{3}\right) \\
= & \int d^{3} k_{1} e^{-i \mathbf{k}_{1} \cdot \mathbf{x}_{13}} \int d^{3} k_{2} e^{-i \mathbf{k}_{2} \cdot \mathbf{x}_{23}} B\left(\mathbf{k}_{1}, \mathbf{k}_{2}\right) .
\end{aligned}
$$

Here, $\mathbf{x}_{1}, \mathbf{x}_{2}, \mathbf{x}_{3}$ denote the vertices of the triangle;

$$
\mathbf{x}_{i j} \equiv \mathbf{x}_{i}-\mathbf{x}_{j}, \quad x_{i j} \equiv\left|\mathbf{x}_{i}-\mathbf{x}_{j}\right|
$$

and $\delta_{D}\left(\mathbf{k}_{123}\right)$ is the Dirac delta function for the sum of the three wavevectors $\mathbf{k}_{123}=\mathbf{k}_{1}+\mathbf{k}_{2}+\mathbf{k}_{3}$.

A simple expression for $\zeta\left(\mathbf{x}_{1}, \mathbf{x}_{2}, \mathbf{x}_{3}\right)$ has been obtained by Jing and Börner [48] assuming the tree-level matter bispectrum given in equation (1),

$$
\begin{aligned}
\zeta= & \frac{10}{7} \xi\left(x_{12}\right) \xi\left(x_{13}\right) \\
& -\left[\eta_{2}\left(x_{12}\right) \eta_{0}\left(x_{13}\right)+\eta_{0}\left(x_{12}\right) \eta_{2}\left(x_{13}\right)\right] \mathbf{x}_{12} \cdot \mathbf{x}_{13} \\
& +\frac{4}{7}\left[\epsilon\left(x_{12}\right) \epsilon\left(x_{13}\right)\left(\mathbf{x}_{12} \cdot \mathbf{x}_{13}\right)^{2}\right. \\
& +\eta_{2}\left(x_{13}\right) \epsilon\left(x_{12}\right) x_{12}^{2}+\eta_{2}\left(x_{12}\right) \epsilon\left(x_{13}\right) x_{13}^{2} \\
& \left.+3 \eta_{2}\left(x_{12}\right) \eta_{2}\left(x_{13}\right)\right]+2 \text { perm. }
\end{aligned}
$$

where the auxiliary functions $\eta_{l}(x)$ and $\epsilon(x)$ are defined in Appendix A. They are related to derivatives of the two-point correlation function.

The matter $3 \mathrm{PCF}$ is usually measured using survey data, under the assumption that galaxy overdensities trace matter overdensities. A simple, local model for the galaxy bias, consistent with the perturbative treatment of dark matter evolution, is given by the expansion in terms of the matter overdensity $\delta(\mathbf{x})[7]$, relating (up to second order) the galaxy overdensity $\delta_{g}(\mathbf{x})$ to $\delta(\mathbf{x})$ as

$$
\delta_{g}(\mathbf{x}) \simeq b_{1} \delta(\mathbf{x})+\frac{b_{2}}{2} \delta^{2}(\mathbf{x})
$$

where the two constants $b_{1}$ and $b_{2}$ are the linear and quadratic bias parameters, respectively. In order to be 
consistent with the PT expression for the dark matter bispectrum it is necessary to retain contributions proportional to $b_{2}$. This is because the galaxy $3 \mathrm{PCF}$ receives two contributions of the same order: the first one from the dark matter, tree-level, 3PCF and the second one from the non-linear bias parameter $b_{2}$. From equation (6) one obtains

$$
\begin{aligned}
\left\langle\delta_{g}\left(\mathbf{x}_{1}\right) \delta_{g}\left(\mathbf{x}_{2}\right) \delta_{g}\left(\mathbf{x}_{3}\right)\right\rangle & =b_{1}^{3} \zeta\left(\mathbf{x}_{1}, \mathbf{x}_{2}, \mathbf{x}_{3}\right) \\
& +b_{1}^{2} b_{2}\left[\xi_{12} \xi_{13}+2 \text { perm. }\right],
\end{aligned}
$$

where $\zeta\left(\mathbf{x}_{1}, \mathbf{x}_{2}, \mathbf{x}_{3}\right)$ is the matter $3 \mathrm{PCF}$ and $\xi_{i j}$ is a shorthand notation for the matter two-point function for a given separation

$$
\xi_{i j} \equiv \xi\left(x_{i j}\right)=\left\langle\delta\left(\mathbf{x}_{i}\right) \delta\left(\mathbf{x}_{j}\right)\right\rangle .
$$

\section{B. Magnification Corrections}

Due to the effect of weak lensing magnification, the observed galaxy number density $n(<m, \hat{n})$ below a certain limiting magnitude $m$ in a given direction $\hat{n}$ in the sky is not equal to the actual density $n_{0}$, but is related to it via [49]

$$
n(<m, \hat{n})=n_{0}(<m, \hat{n}) \mu(\hat{n})^{2.5 s-1},
$$

where $n_{0}=\bar{n}\left[1+\delta_{g}(\hat{n})\right], \mu(\hat{n})$ is the magnification, and $s$ is the slope of the number density as a function of the magnitude $m$

$$
s \equiv \frac{d \ln \left[n_{0}(m)\right]}{d m} .
$$

The magnification $\mu$ is given by

$$
\mu(\hat{n})=\frac{1}{(1-\kappa)^{2}-\gamma^{2}},
$$

where $\kappa(\mathbf{x})$ and $\gamma(\mathbf{x})$ represent, respectively, the convergence and shear (see equations (32) and (33) and appendix $\mathrm{B}$ for the definitions). Following the perturbative approach adopted for the estimation of the large-scale 3 -point correlation function, and using the fact that $\kappa$ and $\gamma$ are linear in the matter density $\delta$, we expand the magnification factor in equation (11) to second order as

$$
\mu \simeq 1+2 \kappa+3 \kappa^{2}+\gamma^{2}
$$

From equations $(9,12)$, the observed galaxy number density can then be expressed as

$$
\begin{aligned}
n(<m, \hat{n}) \simeq & \bar{n}(<m)\left(1+b_{1} \delta+\frac{b_{2}}{2} \delta^{2}\right) \\
& \times\left(1+c_{1} \kappa+\frac{1}{2} c_{2} \kappa^{2}+\frac{1}{2} c_{1} \gamma^{2}\right),
\end{aligned}
$$

where we have defined the constants $c_{1} \equiv(5 s-2)$ and $c_{2} \equiv c_{1}\left(c_{1}+1\right)=(5 s-2)(5 s-1)$. Up to second order in $\delta$, the observed galaxy overdensity is then

$$
\begin{aligned}
\delta_{o b s}(\mathbf{x}) \simeq & b_{1} \delta+c_{1} \kappa+\frac{1}{2} b_{2} \delta^{2}+b_{1} c_{1} \delta \kappa \\
& +\frac{1}{2} c_{2} \kappa^{2}+\frac{1}{2} c_{1} \gamma^{2} .
\end{aligned}
$$

From this expression one derives sixteen different contributions to the observed $3 \mathrm{PCF}$, all corresponding to terms of the same order in perturbation theory. Making use of the short-hand notation $\delta_{i} \equiv \delta\left(\mathbf{x}_{i}\right), \kappa_{i} \equiv \kappa\left(\mathbf{x}_{i}\right)$ and $\gamma_{i} \equiv \gamma\left(\mathbf{x}_{i}\right)$, we have

$$
\begin{aligned}
\left\langle\delta_{\text {obs }}\left(\mathbf{x}_{1}\right) \delta_{\text {obs }}\left(\mathbf{x}_{2}\right) \delta_{\text {obs }}\left(\mathbf{x}_{3}\right)\right\rangle= & b_{1}^{3}\left\langle\delta_{1} \delta_{2} \delta_{3}\right\rangle+b_{1}^{2} b_{2}\left[\frac{1}{2}\left\langle\delta_{1} \delta_{2} \delta_{3}^{2}\right\rangle+2 \text { perm. }\right] \\
& +b_{1}^{2} c_{1}\left[\left\langle\delta_{1} \delta_{2} \kappa_{3}\right\rangle+2 \text { perm. }\right]+b_{1} b_{2} c_{1}\left[\frac{1}{2}\left\langle\delta_{1} \delta_{2}^{2} \kappa_{3}\right\rangle+5 \text { perm. }\right] \\
& +b_{1}^{3} c_{1}\left[\left\langle\delta_{1} \delta_{2} \delta_{3} \kappa_{3}\right\rangle+2 \text { perm. }\right]+b_{1}^{2} c_{2}\left[\frac{1}{2}\left\langle\delta_{1} \delta_{2} \kappa_{3}^{2}\right\rangle+2 \text { perm. }\right] \\
& +b_{1}^{2} c_{1}\left[\frac{1}{2}\left\langle\delta_{1} \delta_{2} \gamma_{3}^{2}\right\rangle+2 \text { perm. }\right]+b_{1} c_{1}^{2}\left[\left\langle\delta_{1} \kappa_{2} \kappa_{3}\right\rangle+2 \text { perm. }\right] \\
& +b_{2} c_{1}^{2}\left[\frac{1}{2}\left\langle\delta_{1}^{2} \kappa_{2} \kappa_{3}\right\rangle+2 \text { perm. }\right]+b_{1}^{2} c_{1}^{2}\left[\left\langle\delta_{1} \delta_{2} \kappa_{2} \kappa_{3}\right\rangle+5 \text { perm. }\right] \\
& +b_{1} c_{1} c_{2}\left[\frac{1}{2}\left\langle\delta_{1} \kappa_{2} \kappa_{3}^{2}\right\rangle+5 \text { perm. }\right]+b_{1} c_{1}^{2}\left[\frac{1}{2}\left\langle\delta_{1} \kappa_{2} \gamma_{3}^{2}\right\rangle+5 \text { perm. }\right] \\
& +c_{1}^{3}\left\langle\kappa_{1} \kappa_{2} \kappa_{3}\right\rangle+c_{1}^{2} c_{2}\left[\frac{1}{2}\left\langle\kappa_{1} \kappa_{2} \kappa_{3}^{2}\right\rangle+2 \text { perm. }\right] \\
& +c_{1}^{3}\left[\frac{1}{2}\left\langle\kappa_{1} \kappa_{2} \gamma_{3}^{2}\right\rangle+2 \text { perm. }\right]+b_{1} c_{1}^{3}\left[\left\langle\delta_{1} \kappa_{1} \kappa_{2} \kappa_{3}\right\rangle+2 \text { perm. }\right] .
\end{aligned}
$$


Each of the contributions may involve several permutations of vertices. Note that, as one takes into account second order terms in the convergence, one cannot distinguish unambiguously between "galaxy-galaxylens", "galaxy-lens-lens", and "lens-lens-lens" contribu- tions. For practical purposes only, we will nevertheless identify four groups of terms, the first, referred to as the "galaxy-galaxy-galaxy" group (or GGG), being given by the intrinsic matter $3 \mathrm{PCF}$ plus contributions due to nonlinear bias:

$$
\begin{aligned}
b_{1}^{3}\left\langle\delta_{1} \delta_{2} \delta_{3}\right\rangle & =b_{1}^{3} \zeta\left(\mathbf{x}_{1}, \mathbf{x}_{2}, \mathbf{x}_{3}\right) \quad[\mathrm{GGG}-\mathrm{A}], \\
b_{1}^{2} b_{2}\left[\frac{1}{2}\left\langle\delta_{1} \delta_{2} \delta_{3}^{2}\right\rangle+2 \text { perm. }\right] & =b_{1}^{2} b_{2}\left(\xi_{12} \xi_{13}+\xi_{12} \xi_{23}+\xi_{13} \xi_{23}\right) \quad[\mathrm{GGG}-\mathrm{B}] .
\end{aligned}
$$

For the rest of the paper, we assume without loss of generality that $\chi_{1} \leq \chi_{2} \leq \chi_{3}$, where $\chi_{i}$ represents the comoving distance to the point $\mathbf{x}_{i}$. In the context of the Limber approximation, we can then neglect all terms where lenses in the direction of closer vertices are correlated with overdensities further away, such as $\left\langle\kappa_{1} \delta_{2}\right\rangle$, $\left\langle\kappa_{1} \kappa_{2} \delta_{3}\right\rangle$, and so on. The applicability and limitations of the Limber approximation in this context are discussed in Appendix C.

We can now express the non-vanishing contributions due to lensing magnification as follows, distinguishing a "galaxy-galaxy-lens" (GGL) group,

$$
\begin{array}{rlrl}
b_{1}^{2} c_{1}\left[\left\langle\delta_{1} \delta_{2} \kappa_{3}\right\rangle+2 \text { perm. }\right] & =b_{1}^{2} c_{1}\left\langle\delta_{1} \delta_{2} \kappa_{3}\right\rangle \quad[\mathrm{GGL}-\mathrm{A}], & \\
b_{1} b_{2} c_{1}\left[\frac{1}{2}\left\langle\delta_{1} \delta_{2}^{2} \kappa_{3}\right\rangle+5 \text { perm. }\right] & =b_{1} b_{2} c_{1}\left[\xi_{12}\left(\left\langle\delta_{2} \kappa_{3}\right\rangle+\left\langle\delta_{1} \kappa_{3}\right\rangle\right)+\xi_{13}\left\langle\delta_{1} \kappa_{2}\right\rangle\right] & & {[\mathrm{GGL}-\mathrm{B}],} \\
b_{1}^{3} c_{1}\left[\left\langle\delta_{1} \delta_{2} \delta_{3} \kappa_{3}\right\rangle+2 \text { perm. }\right] & =b_{1}^{3} c_{1}\left(\xi_{23}\left\langle\delta_{1} \kappa_{3}\right\rangle+\xi_{13}\left\langle\delta_{2} \kappa_{3}\right\rangle+\xi_{23}\left\langle\delta_{1} \kappa_{2}\right\rangle\right) & {[\mathrm{GGL}-\mathrm{C}],} \\
b_{1}^{2} c_{2}\left[\frac{1}{2}\left\langle\delta_{1} \delta_{2} \kappa_{3}^{2}\right\rangle+2 \text { perm. }\right] & =b_{1}^{2} c_{2}\left\langle\delta_{1} \kappa_{3}\right\rangle\left\langle\delta_{2} \kappa_{3}\right\rangle & {[\mathrm{GGL}-\mathrm{D}],} \\
b_{1}^{2} c_{1}\left[\frac{1}{2}\left\langle\delta_{1} \delta_{2} \gamma_{3}^{2}\right\rangle+2 \text { perm. }\right] & =b_{1}^{2} c_{1}\left\langle\delta_{1} \gamma_{3}\right\rangle\left\langle\delta_{2} \gamma_{3}\right\rangle \quad[\mathrm{GGL}-\mathrm{E}],
\end{array}
$$

a "galaxy-lens-lens" (GLL) group,

$$
\begin{aligned}
b_{1} c_{1}^{2}\left[\left\langle\delta_{1} \kappa_{2} \kappa_{3}\right\rangle+2 \text { perm. }\right]= & b_{1} c_{1}^{2}\left\langle\delta_{1} \kappa_{2} \kappa_{3}\right\rangle \quad[\mathrm{GLL}-\mathrm{A}], \\
b_{2} c_{1}^{2}\left[\frac{1}{2}\left\langle\delta_{1}^{2} \kappa_{2} \kappa_{3}\right\rangle+2 \text { perm. }\right]= & b_{2} c_{1}^{2}\left\langle\delta_{1} \kappa_{2}\right\rangle\left\langle\delta_{1} \kappa_{3}\right\rangle \quad[\mathrm{GLL}-\mathrm{B}], \\
b_{1}^{2} c_{1}^{2}\left[\left\langle\delta_{1} \delta_{2} \kappa_{2} \kappa_{3}\right\rangle+5 \text { perm. }\right]= & b_{1}^{2} c_{1}^{2}\left[\left\langle\delta_{1} \kappa_{2}\right\rangle\left\langle\delta_{2} \kappa_{3}\right\rangle+\left(\xi_{12}+\xi_{13}\right)\left\langle\kappa_{2} \kappa_{3}\right\rangle\right. \\
& \left.+\left(\xi_{12}+\xi_{23}\right)\left\langle\kappa_{1} \kappa_{3}\right\rangle+\left(\xi_{13}+\xi_{23}\right)\left\langle\kappa_{1} \kappa_{2}\right\rangle\right] \quad[\mathrm{GLL}-\mathrm{C}], \\
b_{1} c_{1} c_{2}\left[\frac{1}{2}\left\langle\delta_{1} \kappa_{2} \kappa_{3}^{2}\right\rangle+5 \text { perm. }\right]= & b_{1} c_{1} c_{2}\left[\left(\left\langle\delta_{1} \kappa_{3}\right\rangle+\left\langle\delta_{1} \kappa_{2}\right\rangle\right)\left\langle\kappa_{2} \kappa_{3}\right\rangle+\left\langle\delta_{2} \kappa_{3}\right\rangle\left\langle\kappa_{1} \kappa_{3}\right\rangle\right] \quad[\mathrm{GLL}-\mathrm{D}], \\
b_{1} c_{1}^{2}\left[\frac{1}{2}\left\langle\delta_{1} \kappa_{2} \gamma_{3}^{2}\right\rangle+5 \text { perm. }\right]= & b_{1} c_{1}^{2}\left[\left\langle\delta_{1} \gamma_{3}\right\rangle\left\langle\kappa_{2} \gamma_{3}\right\rangle+\left\langle\delta_{1} \gamma_{2}\right\rangle\left\langle\gamma_{2} \kappa_{3}\right\rangle+\left\langle\delta_{2} \gamma_{3}\right\rangle\left\langle\kappa_{1} \gamma_{3}\right\rangle\right] \quad[\mathrm{GLL}-\mathrm{E}],
\end{aligned}
$$

and a "lens-lens-lens" (LLL) group,

$$
\begin{array}{rlrl}
c_{1}^{3}\left\langle\kappa_{1} \kappa_{2} \kappa_{3}\right\rangle & =c_{1}^{3}\left\langle\kappa_{1} \kappa_{2} \kappa_{3}\right\rangle \quad[\mathrm{LLL}-\mathrm{A}], & \\
c_{1}^{2} c_{2}\left[\frac{1}{2}\left\langle\kappa_{1} \kappa_{2} \kappa_{3}^{2}\right\rangle+2 \text { perm. }\right] & =c_{1}^{2} c_{2}\left(\left\langle\kappa_{1} \kappa_{2}\right\rangle\left\langle\kappa_{1} \kappa_{3}\right\rangle+\left\langle\kappa_{1} \kappa_{2}\right\rangle\left\langle\kappa_{2} \kappa_{3}\right\rangle+\left\langle\kappa_{1} \kappa_{3}\right\rangle\left\langle\kappa_{2} \kappa_{3}\right\rangle\right) \quad[\text { LLL }-\mathrm{B}], \\
c_{1}^{3}\left[\frac{1}{2}\left\langle\kappa_{1} \kappa_{2} \gamma_{3}^{2}\right\rangle+2 \text { perm. }\right] & =c_{1}^{3}\left(\left\langle\gamma_{1} \kappa_{2}\right\rangle\left\langle\gamma_{1} \kappa_{3}\right\rangle+\left\langle\kappa_{1} \gamma_{2}\right\rangle\left\langle\gamma_{2} \kappa_{3}\right\rangle+\left\langle\kappa_{1} \gamma_{3}\right\rangle\left\langle\kappa_{2} \gamma_{3}\right\rangle\right) & \text { [LLL }-\mathrm{C}], \\
b_{1} c_{1}^{3}\left[\left\langle\delta_{1} \kappa_{1} \kappa_{2} \kappa_{3}\right\rangle+2 \text { perm. }\right] & =b_{1} c_{1}^{3}\left(\left\langle\delta_{1} \kappa_{2}\right\rangle\left\langle\kappa_{1} \kappa_{3}\right\rangle+\left\langle\delta_{1} \kappa_{3}\right\rangle\left\langle\kappa_{1} \kappa_{2}\right\rangle+\left\langle\delta_{2} \kappa_{3}\right\rangle\left\langle\kappa_{1} \kappa_{2}\right\rangle\right) & \text { [LLL }-\mathrm{D}] .
\end{array}
$$


The right hand sides in the equations above include explicitly all non-vanishing permutations. Weak lensing and non-linear bias each couple terms which, as "disconnected" terms, would not contribute to the observed 3PCF otherwise [6]. The non-linear bias couples the matter overdensity at a fixed location with itself, see e.g. the GGG-B contribution [equation (17)]. The magnification effect couples a lens along the line of sight to a galaxy with that galaxy, with a "coupling strength" given by $c_{1}=5 s-2$. For example, for the first term of the GGLC contribution [equation (20)], a galaxy at $\mathbf{x}_{1}$ correlating with a lens along the line of sight to the galaxy at $\mathbf{x}_{3}$, and a galaxy at $\mathbf{x}_{2}$ correlating with the one at $\mathbf{x}_{3}$, result in a contribution to the observed galaxy three-point function. In addition, there are mixed terms where one coupling is due to non-linear bias while the other is due to lensing [e.g., GGL-B, equation (19)].

\section{Evaluation of Corrections}

We now proceed to evaluate the terms contributing to the observed $3 \mathrm{PCF}$, equations (16-31). As shown in Appendix B, the magnification and shear perturbations are given as the following line-of-sight integrals:

$$
\begin{aligned}
\kappa\left(\mathbf{x}_{i}\right)= & \frac{3}{2} \Omega_{m} H_{0}^{2} \int_{0}^{\chi_{i}} d \chi \frac{W_{L}\left(\chi_{i}, \chi\right)}{a(\chi)} \delta\left(\mathbf{y}_{i}(\chi) ; \chi\right) \\
\gamma\left(\mathbf{x}_{i}\right)= & \frac{3}{2} \Omega_{m} H_{0}^{2} \int_{0}^{\chi_{i}} d \chi \frac{W_{L}\left(\chi_{i}, \chi\right)}{a(\chi)} \\
& \times \int d^{3} k \frac{k_{1}^{2}-k_{2}^{2}+2 i k_{1} k_{2}}{k^{2}} \delta_{\mathbf{k}}(\chi) e^{-i \mathbf{k} \cdot \mathbf{y}_{i}(\chi)}
\end{aligned}
$$

Here, $W_{L}\left(\chi_{i}, \chi\right)=\chi / \chi_{i}\left(\chi_{i}-\chi\right)$, and $\chi_{i}$ is the distance to $\mathbf{x}_{i}$, while $\chi$ is the lens distance. $\mathbf{y}_{i}(\chi)=\left(\chi / \chi_{i}\right) \mathbf{x}_{i}$ denotes a point moving along the line of sight connecting the observer and the source located at $\mathbf{x}_{i}$ at a distance $\chi_{i}$. In the following, we also use the shorthand notation $C \equiv 3 \Omega_{m} H_{0}^{2} / 2$.

In addition to the matter two-point and three-point functions $\xi$ and $\zeta$, we need to calculate permutations of 3 different connected three-point terms, $\langle\delta \delta \kappa\rangle,\langle\delta \kappa \kappa\rangle$, $\langle\kappa \kappa \kappa\rangle$, and of four different two-point terms, $\langle\delta \kappa\rangle,\langle\delta \gamma\rangle$, $\langle\kappa \kappa\rangle,\langle\kappa \gamma\rangle$. It is quite remarkable that they can all be calculated with the help of only four auxiliary functions, as detailed in Appendix A.

We will make use of the Limber approximation [50] in the evaluation of the magnification terms. Each application of the approximation sets the lens distance $\chi$ in equations (32) and (33) to the value of the observable (e.g., $\delta$ ) correlated with, thereby reducing the number of line-of-sight integrals by one, and fixing the separation $r$ to $r_{\perp}$, the projected (or transverse) separation. See Appendix $\mathrm{C}$ for a discussion.

By defining the "transverse" two- and three-point correlation functions, $\tilde{\xi}$ and $\tilde{\zeta}$, respectively, and the auxiliary function $\tilde{\epsilon}$ (see Appendix A for more details), one obtains very similar expressions for almost all magnification terms. The projected functions are given by

$$
\begin{aligned}
\tilde{\xi}\left(\mathbf{x}_{1}, \mathbf{x}_{2} ; \chi\right) \equiv & (2 \pi)^{2} \int d^{2} k_{\perp} e^{-i \mathbf{k}_{\perp} \cdot\left(\mathbf{x}_{1 \perp}-\mathbf{x}_{2 \perp}\right)} \\
& \times P_{L}\left(k_{\perp} ; \chi\right) \\
\widetilde{\zeta}\left(\mathbf{x}_{1}, \mathbf{x}_{2}, \mathbf{x}_{3} ; \chi\right) \equiv & (2 \pi)^{2} \int d^{2} k_{1 \perp} e^{-i \mathbf{k}_{1 \perp} \cdot\left(\mathbf{x}_{1 \perp}-\mathbf{x}_{3 \perp}\right)} \\
& \times \int d^{2} k_{2 \perp} e^{-i \mathbf{k}_{2 \perp} \cdot\left(\mathbf{x}_{2 \perp}-\mathbf{x}_{3 \perp}\right)} \\
& \times B\left(\mathbf{k}_{1 \perp}, \mathbf{k}_{2 \perp} ; \chi\right)
\end{aligned}
$$

and

$$
\widetilde{\epsilon}(x ; \chi) \equiv \frac{(2 \pi)^{2}}{x^{3}} \int d k P_{L}(k ; \chi)\left[k x J_{0}(k x)-2 J_{1}(k r)\right]
$$

We give all relevant quantities in terms of these functions below, first as the exact integral, then using the Limber approximation (L.A.). In all instances, $x_{i j \perp}$ denotes the transverse component of the separation, i.e. the projection onto the sky plane.

$$
\begin{aligned}
\left\langle\delta\left(\mathbf{x}_{1}\right) \delta\left(\mathbf{x}_{2}\right) \kappa\left(\mathbf{x}_{3}\right)\right\rangle & =C \int_{0}^{\chi_{3}} d \chi \frac{W_{L}\left(\chi_{3}, \chi\right)}{a(\chi)} \zeta\left(\mathbf{x}_{1}, \mathbf{x}_{2}, \mathbf{y}_{3}(\chi)\right) \\
& \stackrel{\text { L.A. }}{\simeq} C \frac{W_{L}\left(\chi_{3}, \chi_{1}\right)}{a\left(\chi_{1}\right)} \widetilde{\zeta}\left(\mathbf{x}_{1 \perp}, \mathbf{x}_{2 \perp}, \mathbf{x}_{3 \perp} ; \chi_{1}\right) \delta_{D}\left(\chi_{1}-\chi_{2}\right), \\
\left\langle\delta\left(\mathbf{x}_{1}\right) \kappa\left(\mathbf{x}_{2}\right) \kappa\left(\mathbf{x}_{3}\right)\right\rangle & =C^{2} \int_{0}^{\chi_{2}} d \chi \frac{W_{L}\left(\chi_{2}, \chi\right)}{a(\chi)} \int_{0}^{\chi_{3}} d \chi^{\prime} \frac{W_{L}\left(\chi_{3}, \chi^{\prime}\right)}{a\left(\chi^{\prime}\right)} \zeta\left(\mathbf{x}_{1}, \mathbf{y}_{2}(\chi), \mathbf{y}_{3}\left(\chi^{\prime}\right)\right) \\
& \stackrel{\text { L.A. }}{\simeq} C^{2} \frac{W_{L}\left(\chi_{2}, \chi_{1}\right) W_{L}\left(\chi_{3}, \chi_{1}\right)}{a^{2}\left(\chi_{1}\right)} \widetilde{\zeta}\left(\mathbf{x}_{1 \perp}, \mathbf{x}_{2 \perp}, \mathbf{x}_{3 \perp} ; \chi_{1}\right),
\end{aligned}
$$




$$
\begin{aligned}
& \left\langle\kappa\left(\mathbf{x}_{1}\right) \kappa\left(\mathbf{x}_{2}\right) \kappa\left(\mathbf{x}_{3}\right)\right\rangle=C^{3} \int_{0}^{\chi_{1}} d \chi \frac{W_{L}\left(\chi_{1}, \chi\right)}{a(\chi)} \int_{0}^{\chi_{2}} d \chi^{\prime} \frac{W_{L}\left(\chi_{2}, \chi^{\prime}\right)}{a\left(\chi^{\prime}\right)} \int_{0}^{\chi_{3}} d \chi^{\prime \prime} \frac{W_{L}\left(\chi_{3}, \chi^{\prime \prime}\right)}{a\left(\chi^{\prime \prime}\right)} \zeta\left(\mathbf{y}_{1}(\chi), \mathbf{y}_{2}\left(\chi^{\prime}\right), \mathbf{y}_{3}\left(\chi^{\prime \prime}\right)\right) \\
& \stackrel{\text { L.A. }}{\simeq} C^{3} \int_{0}^{\chi_{1}} d \chi \frac{W_{L}\left(\chi_{1}, \chi\right) W_{L}\left(\chi_{2}, \chi\right) W_{L}\left(\chi_{3}, \chi\right)}{a^{3}(\chi)} \widetilde{\zeta}\left(\mathbf{y}_{1 \perp}(\chi), \mathbf{y}_{2 \perp}(\chi), \mathbf{y}_{3 \perp}(\chi) ; \chi\right), \\
& \left\langle\delta\left(\mathbf{x}_{1}\right) \kappa\left(\mathbf{x}_{2}\right)\right\rangle=C \int_{0}^{\chi_{2}} d \chi \frac{W_{L}\left(\chi_{2}, \chi\right)}{a(\chi)} \xi\left(\mathbf{x}_{1}, \mathbf{y}_{2}(\chi)\right) \\
& \stackrel{\text { L.A. }}{\simeq} C \frac{W_{L}\left(\chi_{2}, \chi_{1}\right)}{a\left(\chi_{1}\right)} \widetilde{\xi}\left(\mathbf{x}_{1 \perp}, \mathbf{x}_{2 \perp} ; \chi_{1}\right) \\
& \left\langle\kappa\left(\mathbf{x}_{1}\right) \kappa\left(\mathbf{x}_{2}\right)\right\rangle=C^{2} \int_{0}^{\chi_{1}} d \chi \frac{W_{L}\left(\chi_{1}, \chi\right)}{a(\chi)} \int_{0}^{\chi_{2}} d \chi^{\prime} \frac{W_{L}\left(\chi_{2}, \chi^{\prime}\right)}{a\left(\chi^{\prime}\right)} \xi\left(\mathbf{y}_{1}(\chi), \mathbf{y}_{2}\left(\chi^{\prime}\right)\right) \\
& \stackrel{\text { L.A. }}{\simeq} C^{2} \int_{0}^{\chi_{1}} d \chi \frac{W_{L}\left(\chi_{1}, \chi\right) W_{L}\left(\chi_{2}, \chi\right)}{a^{2}(\chi)} \widetilde{\xi}\left(\mathbf{y}_{1 \perp}(\chi), \mathbf{y}_{2 \perp}(\chi) ; \chi\right), \\
& \left\langle\delta\left(\mathbf{x}_{1}\right) \gamma\left(\mathbf{x}_{2}\right)\right\rangle=C \int_{0}^{\chi_{2}} d \chi \frac{W_{L}\left(\chi_{2}, \chi\right)}{a(\chi)} \int d^{3} k P_{L}(k, \chi) \frac{k_{1}^{2}-k_{2}^{2}+2 i k_{1} k_{2}}{k^{2}} e^{-i \mathbf{k} \cdot\left(\mathbf{y}_{2}(\chi)-\mathbf{x}_{1}\right)} \\
& \stackrel{\text { L.A. }}{\simeq} C \frac{W_{L}\left(\chi_{2}, \chi_{1}\right)}{a\left(\chi_{1}\right)} x_{12, \perp}^{2} \widetilde{\epsilon}\left(x_{12, \perp} ; \chi_{1}\right), \\
& \left\langle\kappa\left(\mathbf{x}_{1}\right) \gamma\left(\mathbf{x}_{2}\right)\right\rangle=C^{2} \int_{0}^{\chi_{1}} d \chi \frac{W_{L}\left(\chi_{1}, \chi\right)}{a(\chi)} \int_{0}^{\chi_{2}} d \chi^{\prime} \frac{W_{L}\left(\chi_{2}, \chi^{\prime}\right)}{a\left(\chi^{\prime}\right)} \\
& \times \int d^{3} k P_{L}\left(k ; \chi ; \chi^{\prime}\right) \frac{k_{1}^{2}-k_{2}^{2}+2 i k_{1} k_{2}}{k^{2}} e^{-i \mathbf{k} \cdot\left(\mathbf{y}_{2}\left(\chi^{\prime}\right)-\mathbf{y}_{1}(\chi)\right)} \\
& \stackrel{\text { L.A. }}{\simeq} C^{2} \int_{0}^{\min \left(\chi_{1}, \chi_{2}\right)} d \chi \frac{W_{L}\left(\chi_{1}, \chi\right) W_{L}\left(\chi_{2}, \chi\right)}{a^{2}(\chi)} y_{12, \perp}^{2} \widetilde{\epsilon}\left(y_{12, \perp}(\chi) ; \chi\right) \text {. }
\end{aligned}
$$

A few comments are in order. To gain a better understanding of the effect of applying the Limber approximation, consider equation (38). The first, exact, line requires the integration of the matter three-point function - weighted by the appropriate geometrical lensing factors - over all triangles that have one vertex fixed at $\mathbf{x}_{1}$ while the other two (located at $\mathbf{y}_{2}$ and $\mathbf{y}_{3}$ ) are moving along the lines of sight connecting the observer to the source locations at $\mathbf{x}_{2}$ and $\mathbf{x}_{3}$, respectively. Similarly, in equation (39) we integrate the unlensed $3 \mathrm{PCF}$ over all triangles with vertices $\left(\mathbf{y}_{1}, \mathbf{y}_{2}, \mathbf{y}_{3}\right)$ lying on the three lines of sight connecting the observer to the sources. For all these terms, the Limber approximation counts the largest contributions to the integrals, which arise from configurations that are perpendicular to the line of sight, i.e. where the separation $x_{i j \|}$ along the line of sight is of order $x_{i j \perp}$ or less. This is because the two and three point correlation functions increase as the separations get smaller. The largest contributions to the integrals will therefore arise when the distance between the points $\mathbf{y}_{i}$ moving along the lines of sight are smallest, which corresponds to transverse separation vectors, i.e. perpendicular to the line of sight. Hence, after the Limber approximation all terms are proportional to the "transverse" correlation functions evaluated at the transverse displacements $x_{i \perp}$, $y_{i \perp}$. Clearly, the magnification terms depend on the size and configuration of the projected triangle, rather than the three-dimensional triangle itself.

However, it is important to note that the Limber approximation has limitations. Most severely, for the GGLA term [equation (37)], applying the Limber approximation yields an unphysical $\delta_{D}\left(\chi_{1}-\chi_{2}\right)$, as the lens is forced to be at the same distance $\chi$ as both $\delta_{1}$ and $\delta_{2}$. To avoid this artifact of the approximation, we evaluate the exact integral for this term. For all other terms, we discuss the accuracy of the Limber approximation in Appendix C. Briefly, we found that, whenever an integral over the line of sight remains in the final expression, the Limber approximation is accurate. This is the case for the $\langle\kappa \kappa \kappa\rangle$, $\langle\kappa \kappa\rangle$, and $\langle\kappa \gamma\rangle$ terms. For the other terms, the approximation can become invalid in certain limits.

After specifying the vertices of the triangle, equations (37-43) above then allow a calculation of all the terms contributing to the observed galaxy $3 \mathrm{PCF}$. The results of this calculation are reported in Sec. III. Before plunging into the full calculation of all the terms in equations (16-31), however, it is useful to assess the relative magnitude of the different terms, in order to have a better understanding of which of them provide the most relevant contribution to the observed $3 \mathrm{PCF}$. 


\section{Order of magnitude estimates}

We will give an estimate of the order of magnitude of the various contributions in terms of the matter correlation function $\xi(r)$, or, equivalently, the dimensionless matter power spectrum $\Delta^{2}(k)$, since $\xi(r) \sim \Delta^{2}(1 / r)$ (see also section 3 of [44]).

In general, we consider a single scale $r$ for the triangular configuration, so that we might expect these estimates to be more accurate for equilateral configurations. We let $r_{\perp}$ denote the typical extent of the triangle in the direction transverse to the line-of-sight, while $r_{\|}$represents the longitudinal extent. The relative magnitude of these is related to the orientation of the triangle, with $r_{\perp}=r, r_{\|}=0$ for a triangle in the sky plane, and $r_{\perp} \sim 0, r_{\|} \sim r$ for a triangle perpendicular to the sky plane.

We approximate the matter 3PCF simply by the hierarchical relation $\zeta(r, r, r) \sim \xi^{2}(r)$ so that the $\langle\delta \delta \delta\rangle$ contribution is given by

$$
\zeta_{g g g} \equiv b_{1}^{3}\left\langle\delta_{1} \delta_{2} \delta_{3}\right\rangle \sim b_{1}^{3} \xi^{2}(r)
$$

The contributions due to magnification involve the projected functions $\widetilde{\xi}$ and $\widetilde{\zeta}$ which can be approximated as

$$
\widetilde{\xi}\left(r_{\perp}\right) \sim r_{\perp} \xi\left(r_{\perp}\right) \simeq r_{\perp} \Delta^{2}\left(1 / r_{\perp}\right)
$$

and

$$
\widetilde{\zeta}\left(r_{\perp}\right) \sim r_{\perp}^{2} \xi^{2}\left(r_{\perp}\right) \simeq r_{\perp}^{2}\left[\Delta^{2}\left(1 / r_{\perp}\right)\right]^{2}
$$

Note that $\widetilde{\xi}$ and $\widetilde{\zeta}$, as opposed to $\xi$ and $\zeta$, are not dimensionless, but rather have dimensions of length and length squared, respectively. We use these approximations in equations (37-43). Whenever there is an integral, we pull the correlation functions out, setting $\chi \rightarrow \chi_{1} / 2$, $y_{i \perp} \rightarrow r_{\perp} / 2$, and integrate over the lensing weight functions (which can give appreciable numerical factors). When not integrated over, the lensing weight function is approximated as

$$
W_{L}\left(\chi_{2}, \chi_{1}\right) \simeq \chi_{2}-\chi_{1}=r_{\|}
$$

As an example of this approximation scheme, consider the GLL-A term in equation (23),

$$
\begin{aligned}
b_{1} c_{1}^{2}\left\langle\delta_{1} \kappa_{2} \kappa_{3}\right\rangle & =b_{1} c_{1}^{2} C^{2} \frac{W_{L}\left(\chi_{2}, \chi_{1}\right) W_{L}\left(\chi_{3}, \chi_{1}\right)}{a^{2}\left(\chi_{1}\right)} \widetilde{\zeta} \\
& \sim b_{1} c_{1}^{2} C^{2}(1+z)^{2} r_{\|}^{2} r_{\perp}^{2} \xi^{2}\left(r_{\perp}\right),
\end{aligned}
$$

where the first equality follows from the Limber equation in equation (38) and the second line implements the order of magnitude estimates. Here, $z$ denotes the redshift at which the triangle is located, and the correlation functions are to be evaluated at that redshift. Recall that $C \propto H_{0}^{2}$ and the canonical GGG term is of order $b_{1}^{3} \xi^{2}(r)$, so the ratio of this contribution to the canonical term is

$$
\frac{\mathrm{GLL}-\mathrm{A}}{\mathrm{GGG}-\mathrm{A}} \sim\left(\frac{c_{1}}{b_{1}}(1+z)\left(H_{0} r_{\|}\right)\left(H_{0} r_{\perp}\right) \frac{\xi^{2}\left(r_{\perp}\right)}{\xi^{2}(r)}\right)^{2}
$$

The order-of-magnitude expressions can be used to order the contributions according to significance. Since $H_{0} \chi$ is of order 1 , the main suppressing factors here are terms like $H_{0} r$, while the ratios of correlation functions can enhance the expressions somewhat (since $r_{\perp}<r$ and $\xi$ rises rapidly for small $r$ ). The GLL-A contribution is suppressed by a factor of order $(r / \chi)^{4}$, denoted $O(4)$ below, and so is expected to be very small.

Applying this approximation to all contributions leads to

$$
\begin{aligned}
& \frac{b_{1}^{2} c_{1}\left\langle\delta_{1} \delta_{2} \kappa_{3}\right\rangle}{\zeta_{g g g}} \simeq \frac{c_{1}}{b_{1}}(1+z)\left(H_{0} r_{\|}\right)\left(H_{0} r_{\perp}\right)\left[\frac{\xi\left(r_{\perp}\right)}{\xi(r)}\right]^{2} \quad[\text { GGL }-\mathrm{A} \quad O(2)], \\
& \frac{b_{1} b_{2} c_{1}\left\langle\delta_{1} \delta_{2}^{2} \kappa_{3}\right\rangle}{\zeta_{g g g}} \simeq \frac{b_{2} c_{1}}{b_{1}^{2}}(1+z)\left(H_{0} r_{\|}\right)\left(H_{0} r_{\perp}\right) \frac{\xi\left(r_{\perp}\right)}{\xi(r)} \quad[\mathrm{GGL}-\mathrm{B} \quad O(2)] \\
& \frac{b_{1}^{3} c_{1}\left\langle\delta_{1} \delta_{2} \delta_{3} \kappa_{3}\right\rangle}{\zeta_{g g g}} \simeq c_{1}(1+z)\left(H_{0} r_{\|}\right)\left(H_{0} r_{\perp}\right) \frac{\xi\left(r_{\perp}\right)}{\xi(r)} \quad[\text { GGL }-\mathrm{C} \quad O(2)], \\
& \frac{b_{1}^{2} c_{2}\left\langle\delta_{1} \delta_{2} \kappa_{3}^{2}\right\rangle}{\zeta_{g g g}} \simeq \frac{c_{2}}{b_{1}}(1+z)^{2}\left(H_{0} r_{\|}\right)^{2}\left(H_{0} r_{\perp}\right)^{2}\left[\frac{\xi\left(r_{\perp}\right)}{\xi(r)}\right]^{2} \quad[\mathrm{GGL}-\mathrm{D} \quad O(4)] \\
& \frac{b_{1}^{2} c_{1}\left\langle\delta_{1} \delta_{2} \gamma_{3}^{2}\right\rangle}{\zeta_{g g g}} \simeq \frac{c_{1}}{b_{1}}(1+z)^{2}\left(H_{0} r_{\|}\right)^{2}\left(H_{0} r_{\perp}\right)^{2}\left[\frac{\xi\left(r_{\perp}\right)}{\xi(r)}\right]^{2} \quad[\text { GGL }-\mathrm{E} O(4)], \\
& \frac{b_{1} c_{1}^{2}\left\langle\delta_{1} \kappa_{2} \kappa_{3}\right\rangle}{\zeta_{g g g}} \simeq \frac{c_{1}^{2}}{b_{1}^{2}}(1+z)^{2}\left(H_{0} r_{\|}\right)^{2}\left(H_{0} r_{\perp}\right)^{2}\left[\frac{\xi\left(r_{\perp}\right)}{\xi(r)}\right]^{2} \quad[\mathrm{GLL}-\mathrm{A} \quad O(4)], \\
& \frac{b_{2} c_{1}^{2}\left\langle\delta_{1}^{2} \kappa_{2} \kappa_{3}\right\rangle}{\zeta_{g g g}} \simeq \frac{b_{2} c_{1}^{2}}{b_{1}^{3}}(1+z)^{2}\left(H_{0} r_{\|}\right)^{2}\left(H_{0} r_{\perp}\right)^{2}\left[\frac{\xi\left(r_{\perp}\right)}{\xi(r)}\right]^{2} \quad[\text { GLL }-\mathrm{B} \quad O(4)],
\end{aligned}
$$




$$
\begin{aligned}
& \frac{b_{1}^{2} c_{1}^{2}\left\langle\delta_{1} \delta_{2} \kappa_{2} \kappa_{3}\right\rangle}{\zeta_{g g g}} \simeq \frac{c_{1}^{2}}{b_{1}} \frac{1}{30}(1+z)^{2}\left(H_{0} \chi\right)^{3}\left(H_{0} r_{\perp}\right) \frac{\xi\left(r_{\perp} / 2\right)}{\xi(r)} \quad[\text { GLL }-\mathrm{C} O(1)], \\
& \left.\frac{b_{1} c_{1} c_{2}\left\langle\delta_{1} \kappa_{2} \kappa_{3}^{2}\right\rangle}{\zeta_{g g g}} \simeq \frac{c_{1} c_{2}}{b_{1}^{2}} \frac{1}{30}(1+z)^{3}\left(H_{0} \chi\right)^{3}\left(H_{0} r_{\|}\right)\left(H_{0} r_{\perp}\right)^{2}\left[\frac{\xi\left(r_{\perp} / 2\right)}{\xi(r)}\right]^{2} \quad \text { GLL }-\mathrm{D} \quad O(3)\right], \\
& \left.\frac{b_{1} c_{1}^{2}\left\langle\delta_{1} \kappa_{2} \gamma_{3}^{2}\right\rangle}{\zeta_{\text {ggg }}} \simeq \frac{c_{1}^{2}}{b_{1}^{2}} \frac{1}{30}(1+z)^{3}\left(H_{0} \chi\right)^{3}\left(H_{0} r_{\|}\right)\left(H_{0} r_{\perp}\right)^{2}\left[\frac{\xi\left(r_{\perp} / 2\right)}{\xi(r)}\right]^{2} \quad \text { [GLL }-\mathrm{E} O(3)\right], \\
& \frac{c_{1}^{3}\left\langle\kappa_{1} \kappa_{2} \kappa_{3}\right\rangle}{\zeta_{g g g}} \simeq \frac{c_{1}^{3}}{b_{1}^{3}} \frac{1}{140}(1+z)^{3}\left(H_{0} \chi\right)^{4}\left(H_{0} r_{\perp}\right)^{2}\left[\frac{\xi\left(r_{\perp} / 2\right)}{\xi(r)}\right]^{2} \quad[\mathrm{LLL}-\mathrm{A} \quad O(2)], \\
& \frac{c_{1}^{2} c_{2}\left\langle\kappa_{1} \kappa_{2} \kappa_{3}^{2}\right\rangle}{\zeta_{g g g}} \simeq \frac{c_{1}^{2} c_{2}}{b_{1}^{3}} \frac{1}{30^{2}}(1+z)^{4}\left(H_{0} \chi\right)^{6}\left(H_{0} r_{\perp}\right)^{2}\left[\frac{\xi\left(r_{\perp} / 2\right)}{\xi(r)}\right]^{2} \quad[\operatorname{LLL}-\mathrm{B} \quad O(\text { (2) })], \\
& \frac{c_{1}^{3}\left\langle\kappa_{1} \kappa_{2} \gamma_{3}^{2}\right\rangle}{\zeta_{g g g}} \simeq \frac{c_{1}^{3}}{b_{1}^{3}} \frac{1}{30^{2}}(1+z)^{4}\left(H_{0} \chi\right)^{6}\left(H_{0} r_{\perp}\right)^{2}\left[\frac{\xi\left(r_{\perp} / 2\right)}{\xi(r)}\right]^{2} \quad[\text { LLL }-\mathrm{C} \quad O(2)], \\
& \frac{b_{1} c_{1}^{3}\left\langle\delta_{1} \kappa_{1} \kappa_{2} \kappa_{3}\right\rangle}{\zeta_{g g g}} \simeq \frac{c_{1}^{3}}{b_{1}^{2}} \frac{1}{30}(1+z)^{3}\left(H_{0} \chi\right)^{3}\left(H_{0} r_{\|}\right)\left(H_{0} r_{\perp}\right)^{2}\left[\frac{\xi\left(r_{\perp} / 2\right)}{\xi(r)}\right]^{2} \quad[\operatorname{LLL}-\mathrm{D} \quad O(3)] .
\end{aligned}
$$

The order in powers of $H_{0} r$ is indicated by the symbols $O(n)$ in each line. Comparing with the exact expressions, we have verified that the above expressions correctly reproduce the relative ordering of the different terms. The largest contributions to the magnification corrections of the $3 \mathrm{PCF}$ arise from the $O(1)$ and $O(2)$ terms. Terms of order $O(3)$ and $O(4)$ are significantly suppressed.

The significant GGL and GLL terms do not increase dramatically with redshift, as they scale with $(1+z)$ or $(1+z)^{2}$. The terms belonging to the LLL group on the other hand scale with third and fourth power of the redshift: one expects their weight relative to the other terms to increase with increasing redshift. This is consistent with a similar behavior of the GL and LL magnification terms in the two-point correlation function, as already noticed by [43, 44, 47].

\section{RESULTS}

The relative magnitude of the magnification corrections to the galaxy $3 \mathrm{PCF}$ derived in the previous section is highly dependent on the shape of the specific triangular configuration considered, as well as on its orientation with respect to the line-of-sight. Typically, as shown by the estimates presented above, we expect a large effect for triangles whose projection onto the sky is much smaller than the three-dimensional extent, i.e. elongated triangles oriented along the line-of-sight. Another factor to be taken into account is the specific value for the linear and quadratic bias parameters as well as the number count slope that we can expect for the sample under consideration.

\section{A. Specific triangle configurations}

We will not present here a complete analysis of the detectability of this effect as this is beyond the scope of the present work. We will limit ourselves to consider some specific configurations at different redshifts and compute the relative magnification contributions. In section III C we will comment on the uncertainty of the $3 \mathrm{PCF}$ that one might expect in future high-redshift surveys for these specific triangles.

We consider four classes of triangular configurations. The first three correspond to triangles lying in a plane that contains the line-of-sight. In this case, the projections of the three galaxy positions on the sky plane will be aligned. We fix the transverse separation between the points and vary in different ways their line-of-sight separation. Let us use $r_{i j, \perp}$ to denote the transverse separation between galaxies at positions $\mathbf{x}_{i}$ and $\mathbf{x}_{j}$, while $r_{i j, \|}=\chi_{j}-\chi_{i}$ is the separation along the line of sight.

We carefully chose triangle configurations and scales to ensure that the tree-level perturbation theory approach remains valid. We can assume loop corrections in perturbation theory to be subdominant above scales of the order of $20 h^{-1}$ Mpc. At the same time, while the lensing signal becomes relatively larger at large scales, it will still be difficult to measure the three-point correlation at scales of hundreds of Mpc, even in forthcoming redshift surveys.

For the first triangle configuration ("T1"), schematically represented in the left panel of Fig. 1, we keep $r_{13, \perp}=r_{23, \perp}=20 h^{-1} \mathrm{Mpc}, r_{12, \perp}=40 h^{-1} \mathrm{Mpc}$ and $\chi_{2}-\chi_{1}=20 h^{-1} \mathrm{Mpc}$ and we evaluate the $3 \mathrm{PCF}$ and its magnification corrections as the line-of-sight separation $r \equiv \chi_{3}-\chi_{1}$ of the third galaxy is varied from 20 to $200 h^{-1} \mathrm{Mpc}$. 


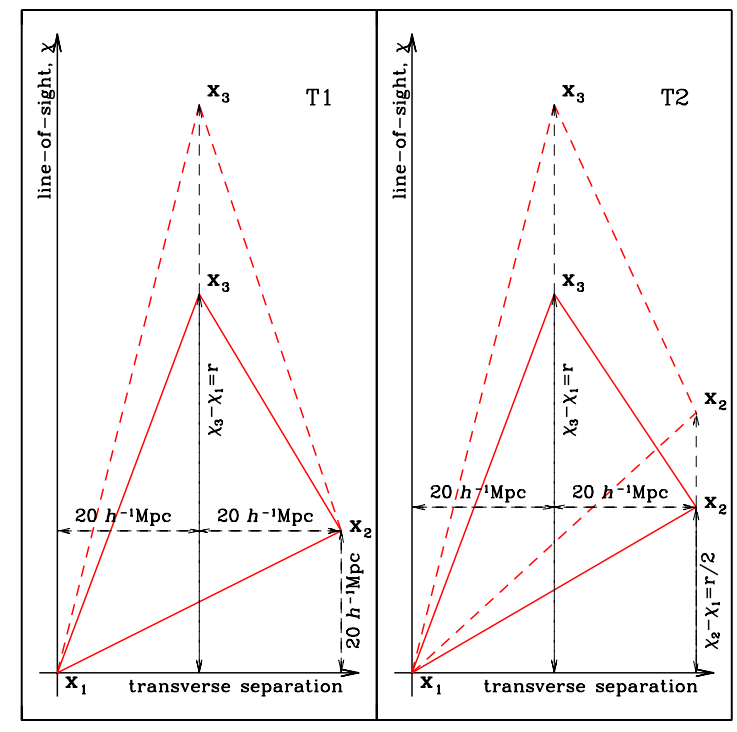

FIG. 1: Triangular configurations T1 and T2. The dashed (red) line shows a second triangle of the same class.

In the second class of configurations ("T2") we keep the same values for the transverse separations as for $\mathrm{T} 1$, but we consider the position $\mathbf{x}_{2}$ to be halfway between $\mathbf{x}_{1}$ and $\mathbf{x}_{3}$ (see Fig. 1, right panel), with $r_{13, \|}$ again varying between 20 and $200 h^{-1} \mathrm{Mpc}$. The third one ("T3") is analogous to T1 but with fixed $r_{23, \|}=20 h^{-1} \mathrm{Mpc}$ and $r_{12, \|}, r_{13, \|}$ varying. All these classes represent a continuous set of triangles starting from an overall size of tens of megaparsec which become progressively elongated and aligned with the line-of-sight as $r_{\|}$increases. From equations (37-43) above, we expect an increasing contribution from magnification for such elongated configurations: while the intrinsic (unlensed) correlation decreases with the triangle size, the lensing terms will remain roughly constant, since they depend on the projected triangle which we keep fixed.

In addition to these three peculiar classes of configurations, we evaluate the lensing contributions for a set of equilateral triangles of increasing size, lying on a plane subtending a $45^{\circ}$ angle with the line-of-sight (see Fig. 2). One can take this to be an example of a triangle with generic orientation, where the parallel and perpendicular separations increase proportionally. The relative lensing contributions are in this case expected to be nearly independent of the side $r$ of the triangle.

Assuming a flat $\Lambda \mathrm{CDM}$ cosmology with parameters: $\Omega_{m}=0.27, \Omega_{b}=0.046, h=0.72, n_{s}=1, \sigma_{8}=0.9$, we evaluate the galaxy $3 \mathrm{PCF}$ at three indicative redshift values: $z=1,3$ and 5 (for definiteness, we refer to them as "low", "intermediate" and "high" redshift in what follows). Specifically, this is assumed to be the redshift of

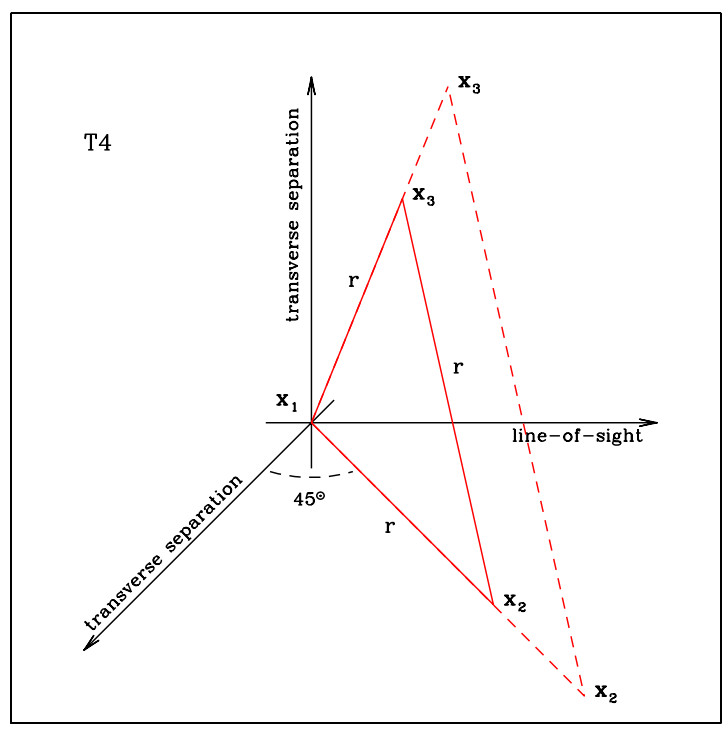

FIG. 2: Triangular configurations T4. The dashed (red) line shows a second triangle of the same class.

the galaxy at location $\mathbf{x}_{1}$, while without loss of generality we always assume that the distances of the three points from the observer obey the condition $\chi_{1} \leq \chi_{2} \leq \chi_{3}$. Small corrections due to the different values of the growth factor at the three locations are easily included since they correspond to multiplicative factors at tree-level in PT. We compute the correlation function $\xi$ and the auxiliary functions $\eta$ and $\epsilon$ from the power spectrum as described in the Appendix, assuming the matter transfer function provided by the CAMB code [51]. Therefore, acoustic features in the power spectrum are included. However, we do not include any smoothing effects due to non-linear gravitational clustering. These are beyond the scope of this paper, and should in any case be quite small at the redshifts considered.

The last ingredient required to evaluate a realistic assessment of the lensing contribution to the measured galaxy $3 \mathrm{PCF}$ is a reasonable estimate of the bias parameters $b_{1}, b_{2}$ and the number count slope $s$. We adopt the expressions for the bias parameters derived in the framework of the Halo Model and of the Halo Occupation Distribution (HOD), $[52,53]$. We will refer in particular to the values computed in [34] for several forthcoming highredshift spectroscopic surveys, where the HOD assumed is the one proposed by [54]. They are derived in terms of the survey mean redshift and expected galaxy density; we assume $n_{g} \simeq 5 \times 10^{-4} h^{3} \mathrm{Mpc}^{-3}$ for the $z=1$ and $z=3$ examples, and $n_{g} \simeq 50 \times 10^{-4} h^{3} \mathrm{Mpc}^{-3}$ for the $z=5$ case. We will also consider values for the survey volume of $V=4 h^{-3} \mathrm{Gpc}^{3}, V=2.7 h^{-3} \mathrm{Gpc}^{3}$, and $V=3.4 h^{-3} \mathrm{Gpc}^{3}$ respectively for low, intermediate and 

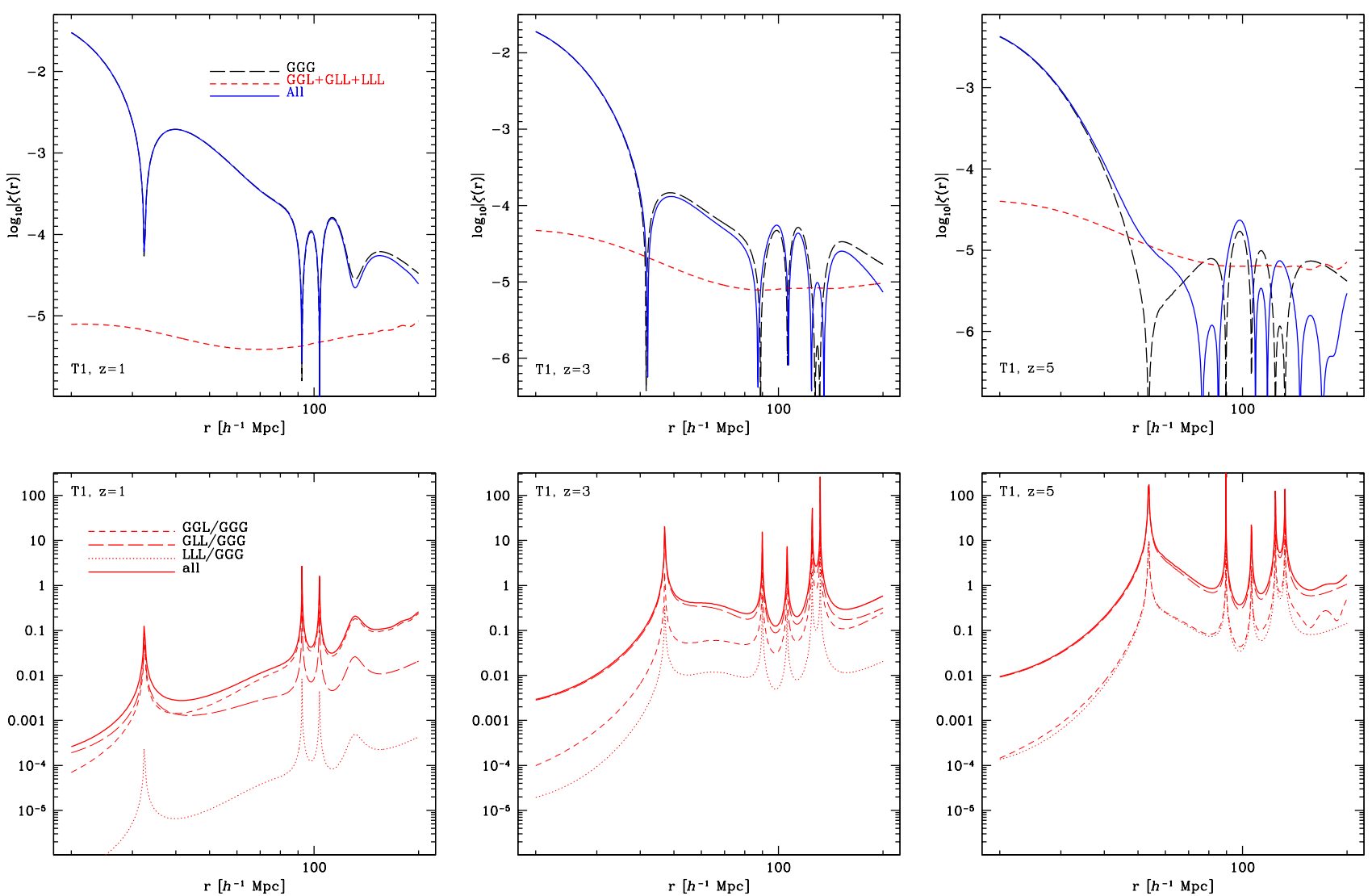

FIG. 3: Triangular configuration T1. The upper panels show the absolute value of the unlensed 3PCF, the GGG group (black, long-dashed line), the contributions due to magnification, sum of the GGL, GLL and LLL terms (red, short-dashed line) and the total (blue, continuous line). The lower panels show the ratio between the magnification contributions and the unlensed 3PCF (continuous line), again as absolute value. We also distinguish the GGL (short-dashed line), GLL (long-dashed line) and LLL terms (dotted line). First, second and third columns correspond respectively to low redshift $(z=1)$, intermediate redshift $(z=3)$, and high redshift $(z=5)$. Specific values for the bias parameters are included as discussed in the text.

high redshift cases, which will be used later for an estimate of the expected error on the 3PCF measurement. These numbers roughly correspond to the characteristics of the proposed surveys WFMOS $(0.5<z<1.3)$, HETDEX $(2<z<4)$, and CIP $(3.5<z<6.5)$. Under these assumptions we obtain bias parameter values of $b_{1} \simeq 2.0$ and $b_{2} \simeq 1.0$ at $z=1, b_{1} \simeq 3.6$ and $b_{2} \simeq 6.2$ at $z=3$ and $b_{1} \simeq 3.7$ and $b_{2} \simeq 6.8$ at $z=5$.

The value of the number count slope $s$ strongly depends on the particular sample, i.e. type of object considered as well as the magnitude cut applied. For galaxies, typical values are in the range $0.2 \lesssim s \lesssim 0.6$ for faint magnitude cuts, and $0.4 \lesssim s \lesssim 1.5$ for bright magnitudes $[44,55]$. For QSO, number count slopes range from $s=-0.2$ to $s=0.8[56,57]$. In contrast to the bias parameters, the number count slope can be inferred from the observed distribution of galaxy apparent magnitudes. Furthermore, by changing the magnitude cut, it is possible to vary $s$ within a limited range [57]. As a fiducial value, we assume $s=0.6$ here, so that $c_{1}=1$ and $c_{2}=2$.

In Fig. 3 (upper panels) we plot the absolute value of the different contributions to the 3PCF for $z=1$, 3 and 5 (left to right column) for the T1 class of triangular configurations. The long-dashed (black) curve represents the unlensed 3PCF, including the non-linear bias contribution, as a function of $r$, i.e. the line-of-sight separation between the points $\mathbf{x}_{1}$ and $\mathbf{x}_{3}$ (see Fig. 1, left panel), while the short-dashed (red) and the continuous (blue) curves represent the magnification contribution (all terms) and the total, lensed 3PCF, respectively. Note that, for this specific configuration, the $3 \mathrm{PCF}$ changes sign at about $r \sim 30 h^{-1} \mathrm{Mpc}$ and several times in the range of scales corresponding to the acoustic features. This is due to the fact that the auxiliary functions $\eta$ and $\epsilon$ in the tree-level PT expression for the 3PCF are related to derivatives of the matter correlation function which exhibits a local minimum and maximum in that range. In the lower panels we plot the ratio of the magnification contributions to the un-lensed 3PCF including the 

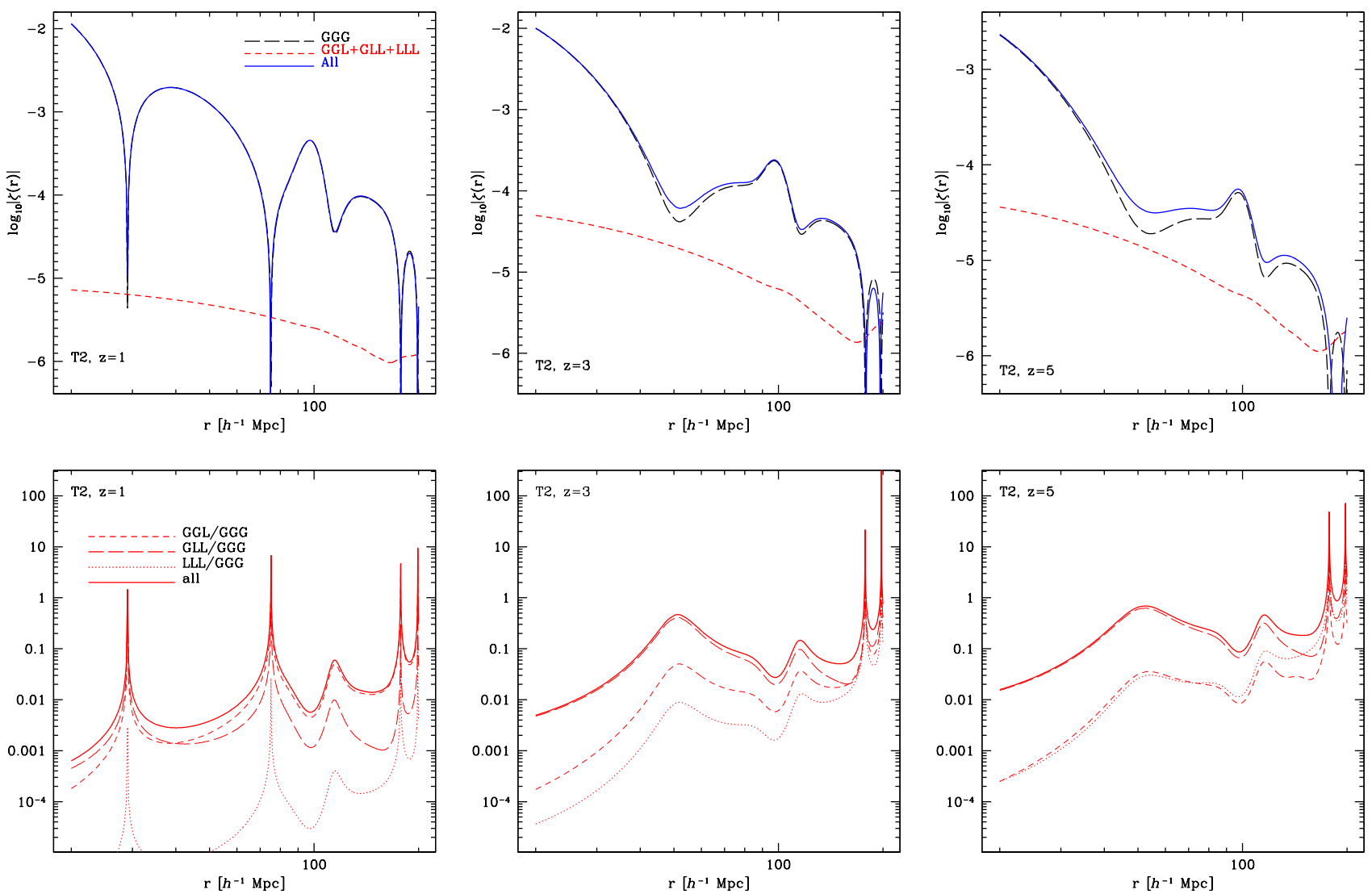

FIG. 4: Triangular configuration T2, $z=1,3$, and 5 .

bias. We also distinguish the contributions of the different groups identified in the previous section, that is the GGL terms (short-dashed curve), the GLL terms (longdashed), and the LLL terms (dotted), while the solid line represents the sum of all of them.

For most of the configurations and redshifts considered, the magnification corrections to the $3 \mathrm{PCF}$ are above the percent level, especially at line-of-sight separations greater than $\sim 50 h^{-1} \mathrm{Mpc}$. Magnification corrections exceed $10 \%$ for separations above $40 h^{-1} \mathrm{Mpc}$ at redshift $z=3$, and above $30 h^{-1}$ Mpc at redshift $z=5$. As mentioned before, these trends are expected: while the magnification terms stay roughly constant, the unlensed $3 \mathrm{PCF}$ is oscillating and decreasing in amplitude. This fact implies that the magnification corrections become more important for increasing separations, and that in the regions where the unlensed $3 \mathrm{PCF}$ changes sign the observed 3PCF will be completely dominated by lensing effects. Furthermore, it is apparent that the magnification effect increases with redshift. This fact has a straightforward physical explanation: the further away the sources are located, the more cosmological structure responsible for lensing is present between the source and the observer, which affects several of the correction terms. Finally, it is also clear from Fig. 3 that while the terms belonging to the GLL group, especially GLL-C, are dominant, the terms belonging to the LLL group have the strongest redshift evolution, and will eventually overtake the other terms at sufficiently high redshift.

In Fig. 4 and 5 the same plots are presented for the triangular configurations T2 and T3, respectively. Most of the comments made about $\mathrm{T} 1$ are also valid for the triangular configurations T2 and T3. At intermediate and high redshifts, the magnification corrections exceed $10 \%$ for separations of $40 \mathrm{~h}^{-1} \mathrm{Mpc}$ and eventually dominate the observed $3 \mathrm{PCF}$. For T2 and T3, the unlensed 3PCF is again oscillating and decreasing in amplitude while the magnification corrections stay roughly constant.

In Fig. 6 we finally present the results for the equilateral configuration T4. In this case, the magnification corrections behave somewhat differently as function of the separation. This is because in this particular case the transverse separation between the sources is not held fixed. Hence, the magnification correction is decreasing with increasing separation roughly in the same way as the overall 3PCF. In this case, then, the lensing contributions to the 3PCF become overwhelming only when the unlensed $3 \mathrm{PCF}$ is changing sign and therefore going through zero. As before, the lensing contributions increase with redshift: at $r_{\|} \gtrsim 40 h^{-1} \mathrm{Mpc}$, they amount 

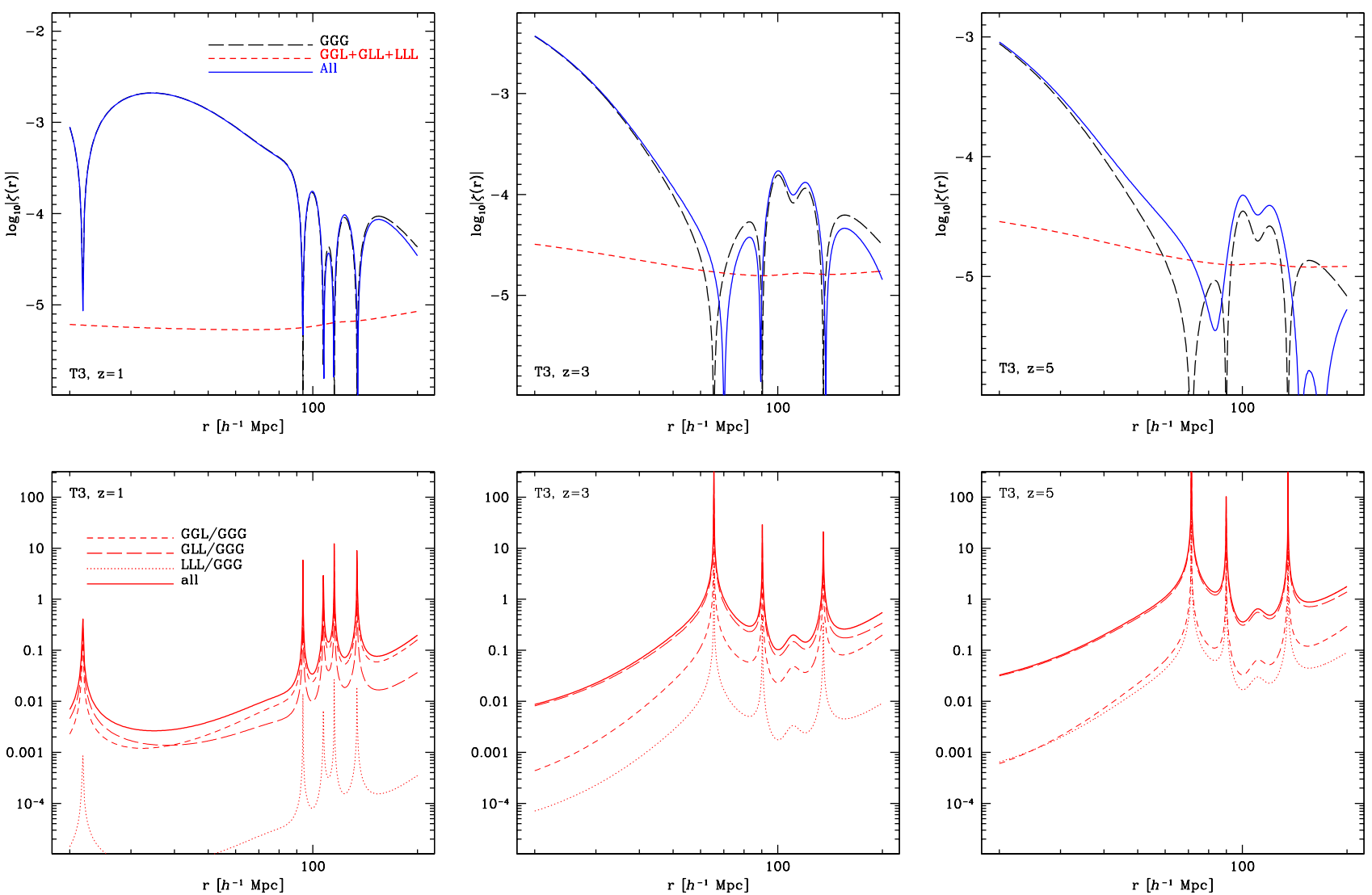

FIG. 5: Triangular configuration T3, $z=1,3$, and 5 .

to less than $1 \%$ for $z=1$, a few percent at $z=3$ and about $10 \%$ at $z=5$.

Going back to equations (18)-(31), it is clear that the magnification terms in different groups depend differently on the value of $s$ (via $c_{1}$ and $c_{2}$ ): the GGL terms scale as $c_{1}$ and $c_{2}$, the GLL terms as $c_{1}^{2}$ and $c_{1} c_{2}$ while the LLL terms as $c_{1}^{3}$ and $c_{1}^{2} c_{2}$. Hence, depending on the value of $s$ of the sample, different groups of terms will dominate. This is shown in Fig. 7, where we show the three sets of contributions as a function of $s$, for a fixed triangle at $z=3$ (the T1 example with $r=60 h^{-1} \mathrm{Mpc}$ ). Note that the GGL and LLL terms are odd with respect to $(s-0.4)$, i.e., they change sign for $s<0.4$, while the GLL terms are even. The dominant individual contribution in this case turns out to be, as expected, the $\left\langle\delta_{1} \delta_{2} \kappa_{2} \kappa_{3}\right\rangle$ (GLLC) term, for all values of $s$. Note that this term increases rapidly with $s$ around the value $s=0.6$ chosen for the plots of Fig. 3-6. Thus, for a sample with high number count slope, the prospects of detection are significantly more optimistic than shown in Fig. 3-6. In the event of a detection, the scaling with $s$ should be observed by measuring the $3 \mathrm{PCF}$ with different apparent magnitude cuts in the survey, corresponding to different values of $s$. In principle, this would allow to disentangle contributions with different scaling behavior, e.g. GLL and LLL terms.

\section{B. Reduced three-point function}

The reduced three-point function is commonly used to measure the non-Gaussianity of the galaxy distribution, as it contains all the information of the full $3 \mathrm{PCF} \zeta$, but is, to first order, independent of the growth and amplitude of matter fluctuations. It is given by

$$
Q\left(\mathbf{x}_{1}, \mathbf{x}_{2}, \mathbf{x}_{3}\right)=\frac{\zeta\left(\mathbf{x}_{1}, \mathbf{x}_{2}, \mathbf{x}_{3}\right)}{\xi_{12} \xi_{13}+\xi_{12} \xi_{23}+\xi_{13} \xi_{23}} .
$$

The reduced bispectrum is defined in an analogous way. In order to exemplify the effects of weak lensing on the reduced $3 \mathrm{PCF}$, we show the intrinsic (unlensed) as well as lensed $Q$ as a function of $\theta=\varangle\left(r_{12}, r_{13}\right)$, the angle at vertex 1 , for a triangle at $z=3$ (Fig. 8). The lensed $Q$ is shown for different orientations of the triangle, specified by the angle $\beta$ between the normal to the plane of the triangle and the line of sight. $\beta$ is chosen so that if $\beta>0$, vertex 3 is further away than 1 and 2 , while it is closer for $\beta<0$. We choose $\mathbf{r}_{12}$ to be perpendicular to the line of sight with $r_{12}=20 h^{-1} \mathrm{Mpc}$, and $r_{13}=80 h^{-1} \mathrm{Mpc}$. For this figure, we chose a value of $s=1.2$ for the number count slope, corresponding to $(5 s-2) / b \approx 1.3$. This value, while high, is not unexpected for bright magnitude cuts (e.g., Fig. 1 in [44]). 

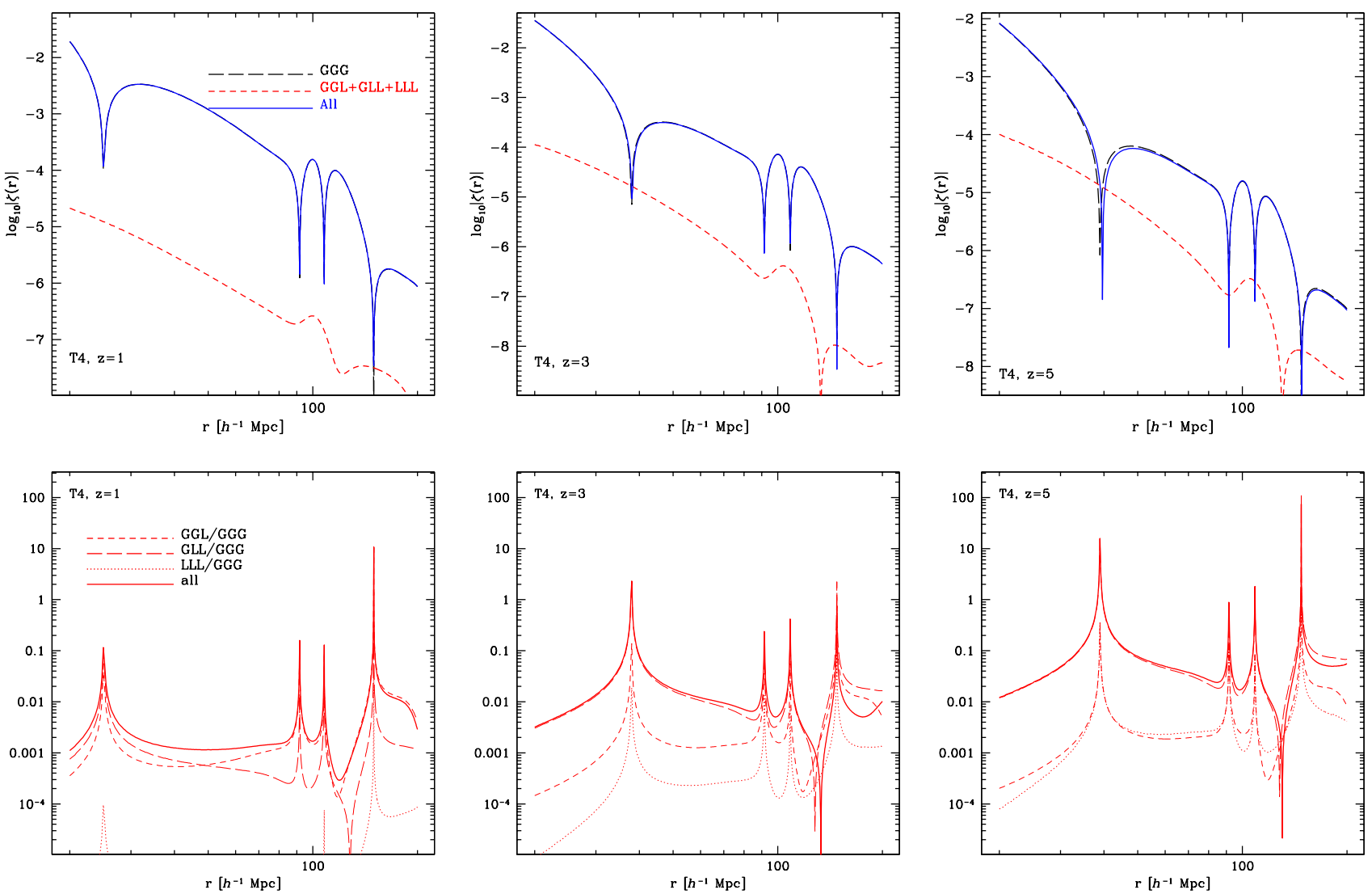

FIG. 6: Triangular configuration T4, $z=1,3$ and 5 .

Following the discussion earlier, we expect the largest lensing contributions when $\beta= \pm \pi / 2$, i.e. when the triangle is oriented along the plane containing the line of sight, since in that case the projected separations are the smallest. In calculating the lensing corrections, we excluded configurations where the perpendicular separation of any two points became less than $10 h^{-1} \mathrm{Mpc}$, since in those cases the perturbation theory approach is expected to break down. This results in the gap in the curve for $\beta=\pi / 2$. In that orientation $r_{13 \perp} \rightarrow 0$ for $\theta=\pi / 2$. As the correlation function rises for $r \rightarrow 0$, we expect the lensing contribution to rise sharply in this range.

Apparently, the lensing effect on $Q$ is appreciable in this configuration. Even for $\beta=0$, i.e. a triangle lying in the sky plane, there is still a finite lensing contribution from the LLL terms. For inclined orientations, GGL and GLL terms contribute significantly. There is an enhancement of $Q$ around $\theta \sim \pi / 2$ where vertex 3 passes behind $(\beta>0)$ or in front of $(\beta<0)$ vertices 1 and 2 . For $\beta$ approaching $+\pi / 2$, there is noticeable structure in the lensing contribution. This is due to the sum over GGL and GLL terms peaking at different values of $\theta$.

Note that the observed $Q$ will be given by the $3 \mathrm{PCF}$ including lensing effects divided by the product of the observed two-point correlation functions which will also include contributions from magnification bias. Since we focus on the lensing effects on the $3 \mathrm{PCF}$, we did not include them here.

\section{Observability of the magnification contribution}

In order to assess the observability of the lensing effects discussed here, we have to estimate the expected statistical uncertainty on the observed three-point function. In Appendix D, we derive a simple and very approximate expression for this uncertainty, assuming correlations are small (as is the case on large scales). To measure the three-point function for a fixed triangle, one defines bins in configuration space, so that for a given galaxy at vertex 1 , triangles are counted for galaxies in the volume $d V_{2}$ around vertex 2 and $d V_{3}$ around vertex 3 . For a survey with $N_{g}$ galaxies with a galaxy volume density of $n_{g}$, we then obtain:

$$
\sigma(\zeta)=N_{g}^{-1 / 2} \sqrt{\frac{1}{n_{g}^{2} d V_{2} d V_{3}}+36} .
$$

For the rough estimates desired here, let us assume a typical bin size of $d V_{2} \sim d V_{3} \sim 10^{4} h^{-3} \mathrm{Mpc}^{3}$, corre- 


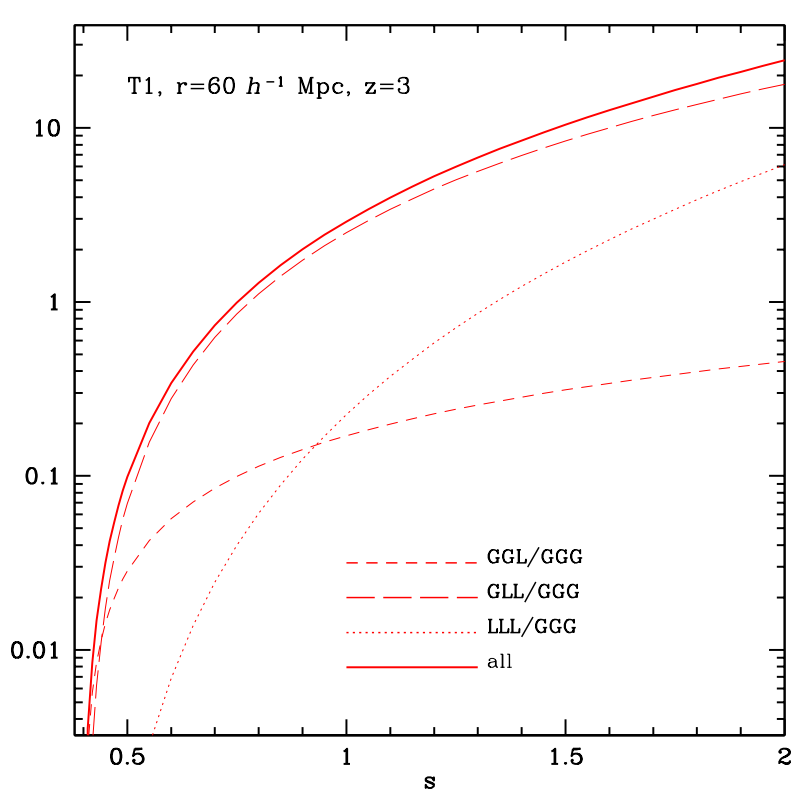

FIG. 7: Ratio (absolute value, as in the lower panels of Figs. 3-6) of the lensing contributions GGL, GLL, and LLL to the unlensed GGG terms, as a function of the number count slope $s$. The triangle, T1 with $r=60 h^{-1} \mathrm{Mpc}$ at $z=3$, is kept fixed.

sponding to a linear dimension of $\sim 20 h^{-1} \mathrm{Mpc}$. Using the values for the galaxy density and volume introduced above for the three ideal surveys at redshifts $z=1$, $z=3$ and $z=5$ we obtain uncertainties on the specific configurations given, respectively, by $\sigma(\zeta) \sim 4 \cdot 10^{-5}$, $\sigma(\zeta) \sim 5 \cdot 10^{-5}$ and $\sigma(\zeta) \sim 1.4 \cdot 10^{-5}$. Note that these uncertainties correspond to triangular configuration bins with a given orientation, so that they can be directly related to the absolute values for the $3 \mathrm{PCF}$ plotted in Fig. 3, 4, 5 and 6 . This suggests that magnification effects should be potentially detectable in the galaxy $3 \mathrm{PCF}$ at redshifts below $z \approx 3$, and clearly measurable at $z \gtrsim 4$.

We expect, on the other hand, that when the correlation function is measured by averaging over all possible orientations for a given triangular shape, the magnification effect will be significantly reduced. However, in this case the expected error will be reduced as well due to the higher statistics. A proper assessment of the magnitude of the correction given a specific $3 \mathrm{PCF}$ estimator is however beyond the scope of this work.

Finally we should mention that another possibility to detect the lensing effects in much smaller surveys is given by cross-correlating galaxies or quasars at different redshifts, or even across different surveys. In this case, there is no intrinsic correlation due to the large line-of-sight separation, while the magnification terms are still significant at small transverse separations. Such a three-point magnification has indeed already been detected in the SDSS [56] in terms of the galaxy-quasar skewness. How-

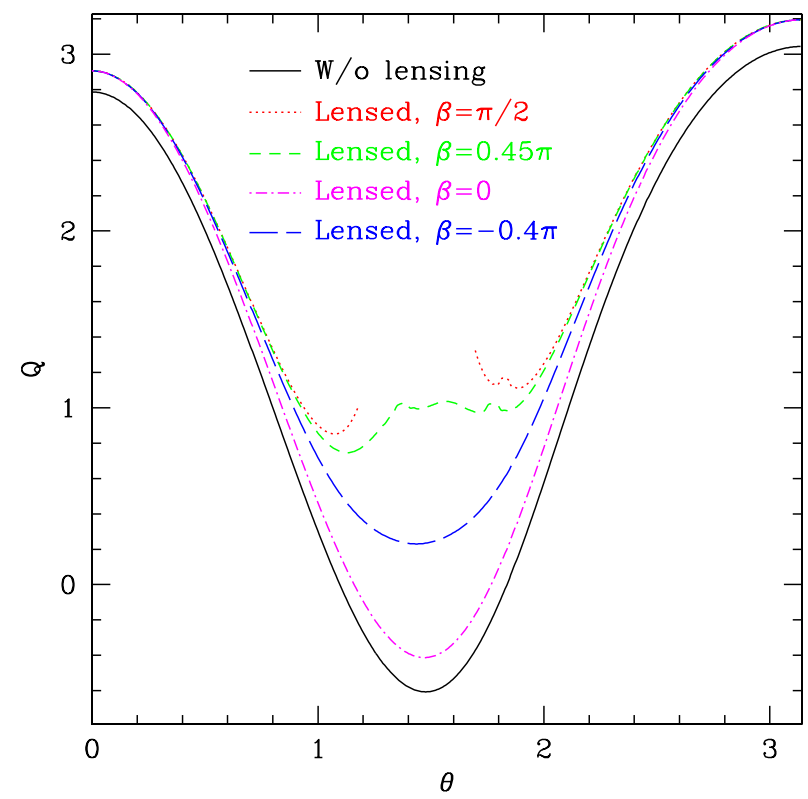

FIG. 8: The reduced three-point function $Q$ as a function of $\theta=\varangle\left(r_{12}, r_{13}\right)$ for a triangle with $r_{12}=20 h^{-1} \mathrm{Mpc}, r_{13}=$ $80 h^{-1} \mathrm{Mpc}$ at $z=3$. The solid (black) curve shows the intrinsic $Q$, while the other curves show the total $Q$ including magnification effects for different orientations of the triangle (see text). We assumed $s=1.2$ for this figure.

ever, no direct comparison between our results and those measurements can be made as we limit ourselves to the correlation function on much larger scales, where perturbation theory is applicable.

\section{CONCLUSIONS}

In this work we analyzed how weak gravitational lensing affects the observed three-point correlation function of galaxies and quasars. As a congruence of photons travels from the source to the observer, the intervening distribution of matter acts by focusing or defocusing it, thus altering the magnitude of the object observed. As a consequence of this effect, any magnitude limited sample and any correlation function measured from it - will be somewhat contaminated by magnification bias.

In a consistent second-order treatment in perturbation theory, weak gravitational lensing contributes 14 different terms. The lensing contribution to the $3 \mathrm{PCF}$ strongly depends on the configuration of the triangle considered, and in particular on its projection on the sky plane. If the projection is held fixed while the triangle is stretched, magnification bias can account for an appreciable fraction of the observed $3 \mathrm{PCF}$ at intermediate to high redshifts. On the other hand, for triangles oriented so that the projection on the sky plane is of similar size as the triangle itself, the magnification corrections to the $3 \mathrm{PCF}$ are 
smaller, reaching percent level at intermediate redshifts and of order $10 \%$ at high redshifts. The lensing contributions are a rapidly varying function of the number count slope $s$ which depends on the sample considered. While we adopted an average value of $s=0.6$ here, the lensing effects increase significantly for larger values of $s$ (e.g., Fig. 7 and 8). A rough estimate of the signal-tonoise suggests that the effect of magnification bias on the galaxy and quasar 3PCF may be detectable by itself at redshift $z \approx 3$ and above.

The results presented here suggest that such magnification corrections need to be taken into account when measurements of non-Gaussianity are carried out using magnitude limited samples from galaxy and quasar catalogs at high redshifts. If neglected, they could in fact bias the determination of the bias parameters $b_{1}$ and $b_{2}$, and affect the sensitivity of galaxy surveys to primordial non-Gaussianity. However, one can also argue that the distinctive dependence of the magnification effects on $s$ as well as $b_{1}, b_{2}$ may be used in the future to unambiguously identify the lensing contribution, and to disentangle the different terms. Ultimately, it might be possible to use the lensing of the $3 \mathrm{PCF}$ as a tool to measure the intervening distribution of matter and the linear growth factor. A more detailed analysis of such aspects will be the focus of forthcoming work.

Since this work represents just a first step in the investigation and the possible exploitation of the lensing of the $3 \mathrm{PCF}$, here we only considered a few specific triangular configurations. However, one would expect that the lensing effects on the projected 3PCF, i.e. the three-point correlation of galaxies in a given redshift bin for a certain projected triangle, will be much larger. This is because many triangles with large line-of-sight separations within the redshift slice will contribute. On the other hand, by counting galaxies in a wide redshift bin, a spectroscopic redshift for each galaxy is no longer necessary, and much larger galaxy statistics become available. A generalization of this is to cross-correlate galaxies from different redshift slices, where the intrinsic correlation is negligible. The lensing effects on the projected $3 \mathrm{PCF}$ will be studied in a future paper.

Also, it would be desirable to extend the modeling of lensing effects to smaller separations, were they are ex- pected and observed to grow rapidly [56]. In principle, one can use non-linear models for the matter power spectrum in the expressions derived here [equations (37)-(43)] to extend the range of validity. However, this is not completely consistent, since the separation into terms such as $\langle\delta \kappa \kappa\rangle,\langle\delta \delta \kappa \kappa\rangle$ is in itself perturbative to leading order in the matter overdensity. This caveat is equally valid for the calculation of magnification effects on the two-point correlation function [43, 44, 47]. A full non-linear calculation of lensing effects on galaxy correlation functions would be considerably more involved and has not been attempted yet.

Finally, the treatment presented here for the magnification bias of the galaxy and quasar $3 \mathrm{PCF}$ is a generalization of the one given in $[43,44,58]$ for the $2 \mathrm{PCF}$. We point out that the perturbation framework used in Sec. II B to derive the magnification corrections is completely general: given enough patience and time, it is straightforward to extend the treatment given here to correlation functions of higher order. One might expect that higher order correlation functions will be more and more affected by magnification bias.

\section{Acknowledgments}

We thank Felipe Marín for useful discussions. E.S. acknowledges the hospitality of the Aspen Center for Physics where part of this work has been completed. This research was supported by the DOE. A.V. thanks Gary Mamon and Jean-Philippe Uzan for useful comments and conversations and thanks the Particle Astrophysics Center of the Fermi National Accelerator Laboratory, where part of this work was carried out, for the hospitality. A.V. is supported by the Agence Nationale pour la Recherche. F.S. thanks the Institute of Physics at Humboldt University, Berlin, for hospitality, and Marek Kowalski, Thomas Lohse, and Ulli Schwanke for inspiring discussions. F.S. was supported by the Kavli Institute for Cosmological Physics at the University of Chicago through grants NSF PHY-0114422 and NSF PHY-0551142 and an endowment from the Kavli Foundation and its founder Fred Kavli.
[1] R. M. Soneira and P. J. E. Peebles, Astrophys. J. 211, 1 (1977).

[2] A. J. Bean, R. S. Ellis, T. Shanks, G. Efstathiou, and B. A. Peterson, Mon. Not. R. Astron. Soc. 205, 605 (1983).

[3] Y. P. Jing, H. J. Mo, and G. Boerner, Astron. Astrophys. 252, 449 (1991).

[4] D. J. Baumgart and J. N. Fry, Astrophys. J. 375, 25 (1991).

[5] J. N. Fry, Astrophys. J. 279, 499 (1984).

[6] F. Bernardeau, S. Colombi, E. Gaztañaga, and R. Scoc- cimarro, Phys. Rep. 367, 1 (2002), astro-ph/0112551.

[7] J. N. Fry and E. Gaztanaga, Astrophys. J. 413, 447 (1993), astro-ph/9302009.

[8] J. N. Fry, Physical Review Letters 73, 215 (1994).

[9] S. Matarrese, L. Verde, and A. F. Heavens, Mon. Not. R. Astron. Soc. 290, 651 (1997), astro-ph/9706059.

[10] R. Scoccimarro, H. A. Feldman, J. N. Fry, and J. A. Frieman, Astrophys. J. 546, 652 (2001), astro-ph/0004087.

[11] E. Sefusatti and R. Scoccimarro, Phys. Rev. D 71, 063001 (2005), astro-ph/0412626.

[12] F. A. Marín, R. H. Wechsler, J. A. Frieman, and R. C. 
Nichol, Astrophys. J. 672, 849 (2008), arXiv:0704.0255.

[13] L. Verde, A. F. Heavens, W. J. Percival, S. Matarrese, C. M. Baugh, J. Bland-Hawthorn, T. Bridges, R. Cannon, S. Cole, M. Colless, et al., Mon. Not. R. Astron. Soc. 335, 432 (2002), astro-ph/0112161.

[14] E. Gaztañaga, P. Norberg, C. M. Baugh, and D. J. Croton, Mon. Not. R. Astron. Soc. 364, 620 (2005), astro$\mathrm{ph} / 0506249$.

[15] J. Pan and I. Szapudi, Mon. Not. R. Astron. Soc. 362, 1363 (2005), astro-ph/0505422.

[16] G. V. Kulkarni, R. C. Nichol, R. K. Sheth, H.-J. Seo, D. J. Eisenstein, and A. Gray, ArXiv Astrophysics eprints (2007), astro-ph/0703340.

[17] T. Nishimichi, I. Kayo, C. Hikage, K. Yahata, A. Taruya, Y. P. Jing, R. K. Sheth, and Y. Suto, ArXiv Astrophysics e-prints (2006), astro-ph/0609740.

[18] R. E. Smith, R. Scoccimarro, and R. K. Sheth, ArXiv Astrophysics e-prints (2006), astro-ph/0609547.

[19] R. E. Smith, R. Scoccimarro, and R. K. Sheth (2007), astro-ph/0703620.

[20] P. McDonald, Phys. Rev. D 74, 103512 (2006), astro$\mathrm{ph} / 0609413$.

[21] E. Sefusatti, M. Crocce, S. Pueblas, and R. Scoccimarro, Phys. Rev. D 74, 023522 (2006), astro-ph/0604505.

[22] J. N. Fry and R. J. Scherrer, Astrophys. J. 429, 36 (1994).

[23] M. J. Chodorowski and F. R. Bouchet, Mon. Not. R. Astron. Soc. 279, 557 (1996), astro-ph/9507038.

[24] R. Scoccimarro, Astrophys. J. 542, 1 (2000), astro$\mathrm{ph} / 0002037$

[25] L. Verde, L. Wang, A. F. Heavens, and M. Kamionkowski, Mon. Not. R. Astron. Soc. 313, 141 (2000), astro-ph/9906301.

[26] R. Durrer, R. Juszkiewicz, M. Kunz, and J.-P. Uzan, Phys. Rev. D 62, 021301(R) (2000), astro-ph/0005087.

[27] R. Scoccimarro, E. Sefusatti, and M. Zaldarriaga, Phys. Rev. D 69, 103513 (2004), astro-ph/0312286.

[28] C. Hikage, E. Komatsu, and T. Matsubara, Astrophys. J. 653, 11 (2006), astro-ph/0607284.

[29] E. Komatsu, A. Kogut, M. R. Nolta, C. L. Bennett, M. Halpern, G. Hinshaw, N. Jarosik, M. Limon, S. S. Meyer, L. Page, et al., Astrophys. J. Suppl. 148, 119 (2003), astro-ph/0302223.

[30] D. N. Spergel, R. Bean, O. Doré, M. R. Nolta, C. L. Bennett, J. Dunkley, G. Hinshaw, N. Jarosik, E. Komatsu, L. Page, et al., ArXiv Astrophysics e-prints (2006), astro$\mathrm{ph} / 0603449$.

[31] P. Creminelli, L. Senatore, M. Zaldarriaga, and M. Tegmark, Journal of Cosmology and Astro-Particle Physics 3, 5 (2007), astro-ph/0610600.

[32] E. Komatsu, J. Dunkley, M. R. Nolta, C. L. Bennett, B. Gold, G. Hinshaw, N. Jarosik, D. Larson, M. Limon, L. Page, et al., ArXiv e-prints 803 (2008), 0803.0547.

[33] A. P. S. Yadav and B. D. Wandelt, ArXiv e-prints 712 (2007), 0712.1148.

[34] E. Sefusatti and E. Komatsu, Phys. Rev. D 76, 083004 (2007), arXiv:0705.0343.

[35] S. Cole, W. J. Percival, J. A. Peacock, P. Norberg, C. M. Baugh, C. S. Frenk, I. Baldry, J. Bland-Hawthorn, T. Bridges, R. Cannon, et al., Mon. Not. R. Astron. Soc. 362, 505 (2005), astro-ph/0501174.

[36] D. J. Eisenstein, I. Zehavi, D. W. Hogg, R. Scoccimarro, M. R. Blanton, R. C. Nichol, R. Scranton, H.-J. Seo,
M. Tegmark, Z. Zheng, et al., Astrophys. J. 633, 560 (2005), astro-ph/0501171.

[37] G. Hütsi, Astron. Astrophys. 449, 891 (2006), astro$\mathrm{ph} / 0512201$.

[38] W. J. Percival, R. C. Nichol, D. J. Eisenstein, D. H. Weinberg, M. Fukugita, A. C. Pope, D. P. Schneider, A. S. Szalay, M. S. Vogeley, I. Zehavi, et al., Astrophys. J. 657, 51 (2007).

[39] G. J. Hill, K. Gebhardt, E. Komatsu, and P. J. MacQueen, in AIP Conf. Proc. 743: The New Cosmology: Conference on Strings and Cosmology, edited by R. E. Allen, D. V. Nanopoulos, and C. N. Pope (2004), pp. 224-233.

[40] K. Glazebrook, D. Eisenstein, A. Dey, B. Nichol, and The WFMOS Feasibility Study Dark Energy Team, ArXiv Astrophysics e-prints (2005), astro-ph/0507457.

[41] URL http://sci.esa.int/euclid.

[42] G. J. Melnick, G. G. Fazio, V. Tolls, D. T. Jaffe, K. Gebhardt, V. Bromm, E. Komatsu, and R. A. Woodruff, in Bulletin of the American Astronomical Society (2004), pp. 1509-+.

[43] A. Vallinotto, S. Dodelson, C. Schimd, and J.-P. Uzan, Phys. Rev. D 75, 103509 (2007), arXiv:astro$\mathrm{ph} / 0702606$.

[44] L. Hui, E. Gaztañaga, and M. LoVerde, Phys. Rev. D 76, 103502 (2007), arXiv:0706.1071.

[45] L. Hui, E. Gaztanaga, and M. LoVerde, ArXiv e-prints 710 (2007), 0710.4191.

[46] M. LoVerde, L. Hui, and E. Gaztañaga, Phys. Rev. D 77, 023512 (2008), arXiv:0708.0031.

[47] T. Matsubara, Astrophys. J. Lett. 537, L77 (2000), arXiv:astro-ph/0004392.

[48] Y. P. Jing and G. Boerner, Astron. Astrophys. 318, 667 (1997), arXiv:astro-ph/9606122.

[49] T. J. Broadhurst, A. N. Taylor, and J. A. Peacock, Astrophys. J. 438, 49 (1995), astro-ph/9406052.

[50] M. Bartelmann and P. Schneider, Phys. Rep. 340, 291 (2001), arXiv:astro-ph/9912508.

[51] A. Lewis, A. Challinor, and A. Lasenby, Astrophys. J. 538, 473 (2000), arXiv:astro-ph/9911177.

[52] H. J. Mo, Y. P. Jing, and S. D. M. White, Mon. Not. R. Astron. Soc. 284, 189 (1997), astro-ph/9603039.

[53] R. Scoccimarro, R. K. Sheth, L. Hui, and B. Jain, Astrophys. J. 546, 20 (2001), astro-ph/0006319.

[54] J. L. Tinker, D. H. Weinberg, Z. Zheng, and I. Zehavi, Astrophys. J. 631, 41 (2005), astro-ph/0411777.

[55] M. LoVerde, L. Hui, and E. Gaztañaga, Phys. Rev. D 75, 043519 (2007).

[56] E. Gaztañaga, Astrophys. J. 589, 82 (2003), arXiv:astro$\mathrm{ph} / 0210311$.

[57] R. Scranton, B. Ménard, G. T. Richards, R. C. Nichol, A. D. Myers, B. Jain, A. Gray, M. Bartelmann, R. J. Brunner, A. J. Connolly, et al., Astrophys. J. 633, 589 (2005), arXiv:astro-ph/0504510.

[58] R. Moessner and B. Jain (1997), astro-ph/9709159.

[59] P. J. E. Peebles, The large-scale structure of the universe (Research supported by the National Science Foundation. Princeton, N.J., Princeton University Press, 1980. 435 p., 1980).

[60] H. J. Mo, Y. P. Jing, and G. Boerner, Astrophys. J. 392, 452 (1992). 


\section{APPENDIX A: MATTER AND GALAXY 3PCF}

\section{Conventions}

In all derivations, we use the following convention for the Fourier transform:

$$
\delta_{\mathbf{k}}=\int \frac{d^{3} x}{(2 \pi)^{3}} e^{i \mathbf{k} \cdot \mathbf{x}} \delta(\mathbf{x}), \quad \delta(\mathbf{x})=\int d^{3} k e^{-i \mathbf{k} \cdot \mathbf{x}} \delta_{\mathbf{k}} .
$$

The power spectrum is defined as

$$
\left\langle\delta_{\mathbf{k}_{1}} \delta_{\mathbf{k}_{2}}\right\rangle \equiv \delta_{D}\left(\mathbf{k}_{1}+\mathbf{k}_{2}\right) P\left(k_{1}\right)
$$

while the bispectrum is defined as

$$
\left\langle\delta_{\mathbf{k}_{1}} \delta_{\mathbf{k}_{2}} \delta_{\mathbf{k}_{3}}\right\rangle \equiv \delta_{D}\left(\mathbf{k}_{1}+\mathbf{k}_{2}+\mathbf{k}_{3}\right) B\left(k_{1}, k_{2}, k_{3}\right) .
$$

The dependence of $P\left(k_{1}\right)$ and of $B\left(k_{1}, k_{2}, k_{3}\right)$ on the redshifts/comoving distances is implicitly understood and omitted throughout the text. Since we are consistently using the linear power spectrum, such dependence can simply be accounted for by multiplying by the appropriate growth factors.

To obtain expressions in the other commonly used Fourier convention, where $\delta(\mathbf{x})=\int d^{3} k /(2 \pi)^{3} \delta_{\mathbf{k}} \exp (i \mathbf{k} \cdot \mathbf{x})$, just replace $P(k)$ with $P(k) /(2 \pi)^{3}$. The same is true for the correlation functions defined below. Further, we again use the notation $\mathbf{x}_{i j}=\mathbf{x}_{i}-\mathbf{x}_{j}, x_{i j}=\left|\mathbf{x}_{i}-\mathbf{x}_{j}\right|$.

\section{Computation of the matter 3PCF - Auxiliary Functions}

The matter two-point correlation function is obtained from the matter power spectrum as

$$
\xi(x)=4 \pi \int d k k^{2} P(k) \frac{\sin (k x)}{k x} .
$$

Assuming the tree-level expression for the bispectrum given in equation (1), Jing and Börner [48] showed that one can write

$$
\begin{aligned}
\zeta\left(\mathbf{x}_{1}, \mathbf{x}_{2}, \mathbf{x}_{3}\right)= & \frac{10}{7} \xi\left(x_{12}\right) \xi\left(x_{13}\right)-\left[\eta_{2}\left(x_{12}\right) \eta_{0}\left(x_{13}\right)+\eta_{0}\left(x_{12}\right) \eta_{2}\left(x_{13}\right)\right] \mathbf{x}_{12} \cdot \mathbf{x}_{13} \\
& +\frac{4}{7}\left[\epsilon\left(x_{12}\right) \epsilon\left(x_{13}\right)\left(\mathbf{x}_{12} \cdot \mathbf{x}_{13}\right)^{2}+\eta_{2}\left(x_{13}\right) \epsilon\left(x_{12}\right) x_{12}^{2}+\eta_{2}\left(x_{12}\right) \epsilon\left(x_{13}\right) x_{13}^{2}+3 \eta_{2}\left(x_{12}\right) \eta_{2}\left(x_{13}\right)\right] \\
& +2 \text { perm. }
\end{aligned}
$$

where

$$
\begin{aligned}
\eta_{l}(x) & \equiv 4 \pi \int d k k^{2} \frac{P(k)}{k^{l}} \frac{k x \cos (k x)-\sin (k x)}{k x^{3}}, \\
\epsilon(x) & \equiv 4 \pi \int d k P(k) \frac{3[\sin (k x)-k x \cos (k x)]-k^{2} x^{2} \sin (k x)}{k x^{5}} .
\end{aligned}
$$

This expression is quite convenient since it allows us to determine the $3 \mathrm{PCF}$ from the functions $\xi, \eta_{l}$ and $\epsilon$ which have to be computed only once. These functions are plotted in Fig. 9.

\section{Transverse $2 \mathrm{PCF}, 3 \mathrm{PCF}$ and auxiliary functions}

When applying the Limber approximation, one can obtain very similar expressions for the magnification contributions in terms of the projected two- and three-point correlation functions, $\widetilde{\xi}$ and $\widetilde{\zeta}$, respecively. The projected two-point function $\widetilde{\xi}$ is defined as the 2-dimensional Fourier transform of the power spectrum in terms of the transverse wavenumber $\mathbf{k}_{\perp}$ (equivalent to forcing $k_{z}=0$ ):

$$
\begin{aligned}
\widetilde{\xi}\left(x_{\perp}\right) & \equiv(2 \pi) \int d^{2} k_{\perp} P\left(k_{\perp}\right) e^{-i \mathbf{k}_{\perp} \cdot \mathbf{x}_{\perp}} \\
& =(2 \pi)^{2} \int d k k P(k) J_{0}\left(k x_{\perp}\right),
\end{aligned}
$$



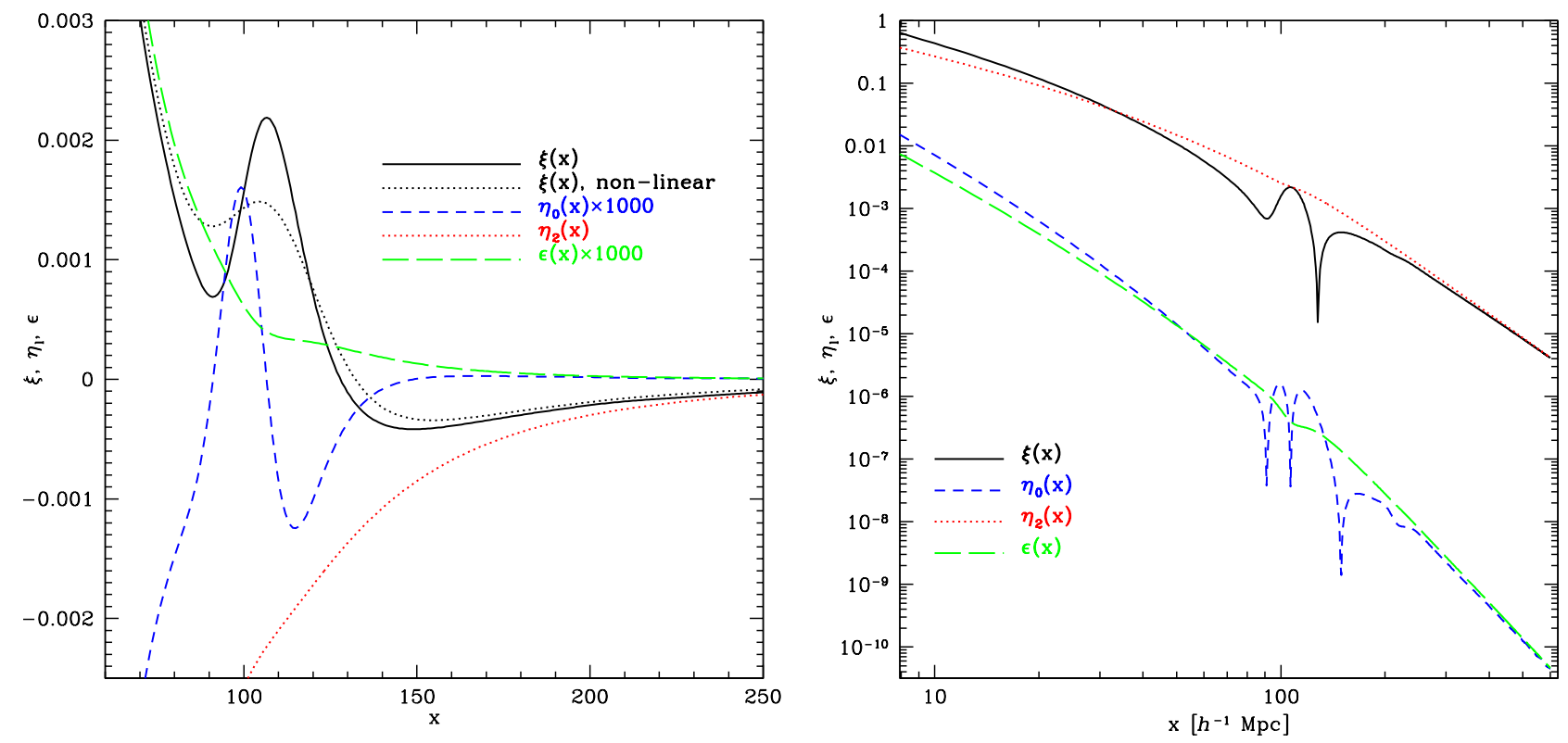

FIG. 9: Linear correlation function $\xi(x)$ and auxiliary functions $\eta_{0}(x), \eta_{2}(x)$ and $\epsilon(x)$ as a function of $x$, evaluated at redshift $z=0$. In the left panel, the non-linear correlation function is also shown. The right panel shows the absolute value of the functions.

where $J_{0}(x)$ is the 0 th order Bessel function. The transverse three-point function $\widetilde{\zeta}$ is defined analogously as

$$
\widetilde{\zeta}\left(\mathbf{x}_{1 \perp}, \mathbf{x}_{2 \perp}, \mathbf{x}_{3 \perp}\right) \equiv(2 \pi)^{2} \int d^{2} k_{1 \perp} e^{-i \mathbf{k}_{1 \perp} \cdot \mathbf{x}_{1 \perp}} \int d^{2} k_{2 \perp} e^{-i \mathbf{k}_{2 \perp} \cdot \mathbf{x}_{2 \perp}} B\left(\mathbf{k}_{1 \perp}, \mathbf{k}_{2 \perp}\right) .
$$

Note that $\widetilde{\xi}$ has dimensions of length, and $\widetilde{\zeta}$ of length squared. In order-of-magnitude terms, $\widetilde{\xi}\left(x_{\perp}\right) \sim x_{\perp} \xi\left(x_{\perp}\right)$, $\widetilde{\zeta}\left(x_{\perp}, x_{\perp}\right) \sim x_{\perp}^{2} \zeta\left(x_{\perp}, x_{\perp}\right)$. In analogy with what is done above for the 3-dimensional 3PCF, one can write

$$
\begin{aligned}
\widetilde{\zeta}\left(\mathbf{x}_{1 \perp}, \mathbf{x}_{2 \perp}, \mathbf{x}_{3 \perp}\right)= & \frac{10}{7} \widetilde{\xi}\left(x_{12 \perp}\right) \widetilde{\xi}\left(x_{13 \perp}\right)-\left[\widetilde{\eta}_{2}\left(x_{12 \perp}\right) \widetilde{\eta}_{0}\left(x_{13 \perp}\right)+\widetilde{\eta}_{0}\left(x_{12 \perp}\right) \widetilde{\eta}_{2}\left(x_{13 \perp}\right)\right] \mathbf{x}_{12 \perp} \cdot \mathbf{x}_{13 \perp} \\
& +\frac{4}{7}\left[\widetilde{\epsilon}\left(x_{12 \perp}\right) \widetilde{\epsilon}\left(x_{13 \perp}\right)\left(\mathbf{x}_{12 \perp} \cdot \mathbf{x}_{13 \perp}\right)^{2}+\widetilde{\eta}_{2}\left(x_{13 \perp}\right) \widetilde{\epsilon}\left(x_{12 \perp}\right) x_{12 \perp}^{2}\right. \\
& \left.+\widetilde{\eta}_{2}\left(x_{12 \perp}\right) \widetilde{\epsilon}\left(x_{13 \perp}\right) x_{13 \perp}^{2}+2 \widetilde{\eta}_{2}\left(x_{12 \perp}\right) \widetilde{\eta}_{2}\left(x_{13 \perp}\right)\right]+2 \text { perm. }
\end{aligned}
$$

where

$$
\begin{aligned}
\widetilde{\eta}_{l}(x) & \equiv \frac{(2 \pi)^{2}}{x} \int d k k^{2} \frac{P(k)}{k^{l}} J_{1}(k x), \\
\widetilde{\epsilon}(x) & \equiv \frac{(2 \pi)^{2}}{x^{3}} \int d k P(k)\left[k x J_{0}(k x)-2 J_{1}(k x)\right] .
\end{aligned}
$$

Here, $J_{l}(x)$ denote Bessel functions of integer order. These functions are plotted in Fig. 10. Since all auxiliary functions are proportional to the linear matter power spectrum, they scale with the square of the matter growth factor. For brevity, we suppress the redshift- or $\chi$-dependence of these functions here and in the following.

\section{APPENDIX B: DERIVATION OF CONVERGENCE AND SHEAR CORRELATORS}

This appendix contains the derivation of convergence $\kappa$ and shear $\gamma$ as well as representative derivations of some of the results shown in equations (37-43). 

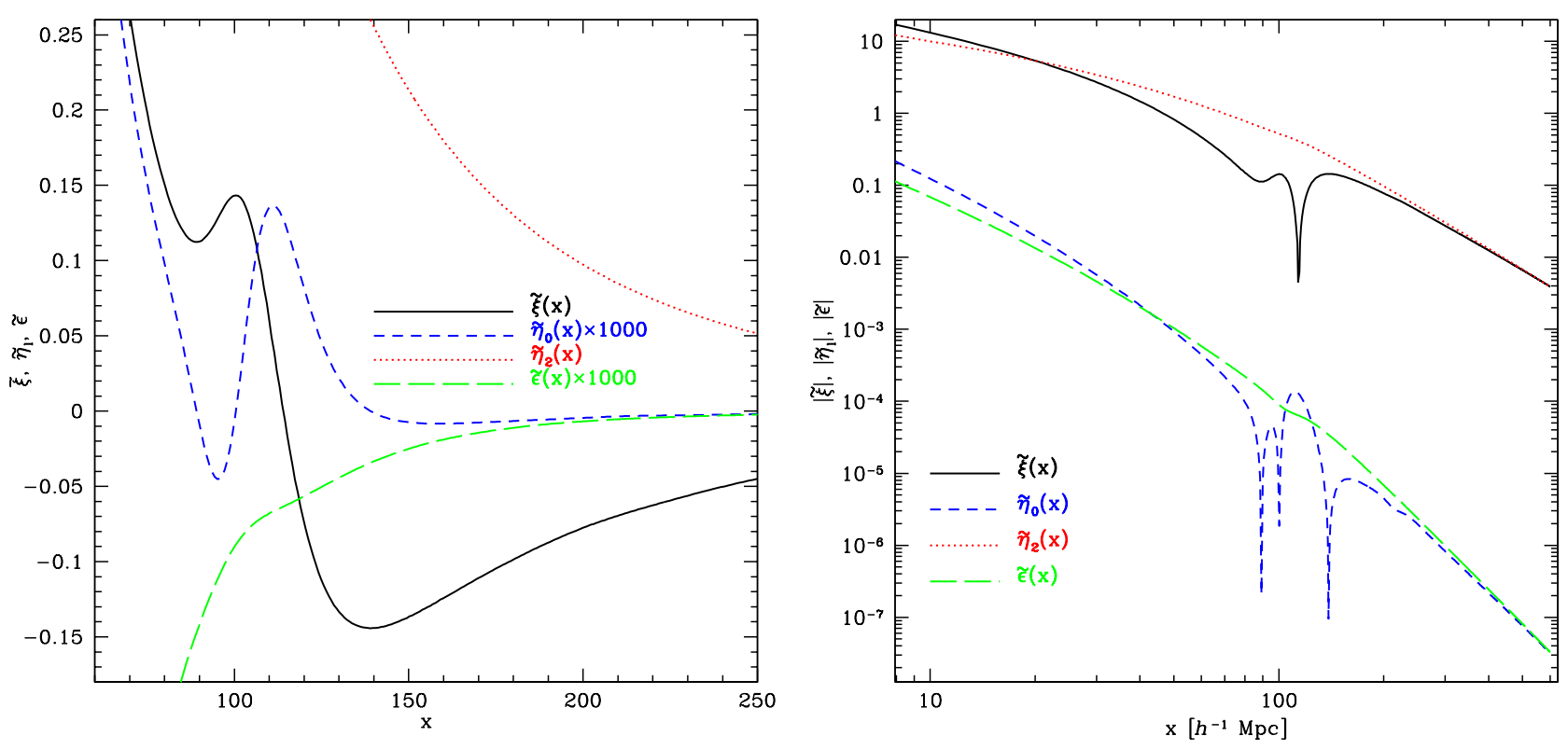

FIG. 10: "Transverse" linear correlation function $\widetilde{\xi}(x)$ and auxiliary functions $\widetilde{\eta}_{0}(x), \widetilde{\eta}_{2}(x)$ and $\widetilde{\epsilon}(x)$ as a function of $x$. The right panel shows the absolute value of the functions.

\section{Convergence and shear}

The projected lensing potential $\hat{\Psi}$ is defined as

$$
\hat{\Psi}(\mathbf{x})=-\int_{0}^{\chi_{s}} d \chi W_{L}\left(\chi_{s}, \chi\right)[\Phi(\mathbf{y}(\chi) ; \chi)-\Psi(\mathbf{y}(\chi) ; \chi)],
$$

where $\Phi, \Psi$ are the gravitational potentials and $\Psi=-\Phi$ at late times in $\Lambda$ CDM and most dark energy models. $\chi_{s}$ is the distance to the source, and $\mathbf{y}(\chi)=\left(\chi / \chi_{s}\right) \mathbf{x}$ parametrizes the line of sight. The convergence $\kappa(\mathbf{x})$ and the complex shear $\gamma(\mathbf{x})$ are then given by

$$
\begin{aligned}
& 2 \kappa=\hat{\Psi}_{, 11}+\hat{\Psi}_{, 22}, \\
& 2 \gamma=\hat{\Psi}_{, 11}-\hat{\Psi}_{, 22}+2 i \hat{\Psi}_{, 12}
\end{aligned}
$$

We now use the Poisson equation,

$$
k^{2} \Phi=-\nabla^{2} \Phi=\frac{3}{2} \Omega_{m} H_{0}^{2} \frac{1}{a} \delta \equiv \frac{C}{a} \delta,
$$

to write $\kappa$ and $\gamma$ in terms of the matter overdensity $\delta$. For the convergence, this yields:

$$
\begin{aligned}
\kappa(\mathbf{x}) & =C \int_{0}^{\chi_{s}} d \chi \frac{W_{L}\left(\chi_{s}, \chi\right)}{a(\chi)} \delta(\mathbf{y}(\chi) ; \chi) \\
& =C \int_{0}^{\chi_{s}} d \chi \frac{W_{L}\left(\chi_{s}, \chi\right)}{a(\chi)} \int d^{3} k e^{-i \mathbf{k} \cdot \mathbf{y}(\chi)} \delta_{\mathbf{k}}(\chi) .
\end{aligned}
$$

For the shear, we obtain:

$$
\gamma(\mathbf{x})=C \int_{0}^{\chi_{s}} d \chi \frac{W_{L}\left(\chi_{s}, \chi\right)}{a(\chi)} \int d^{3} k \frac{k_{1}^{2}-k_{2}^{2}+2 i k_{1} k_{2}}{k^{2}} \delta_{\mathbf{k}}(\chi) e^{-i \mathbf{k} \cdot \mathbf{y}(\chi)}
$$




\section{Derivation of correlators}

As shown in section II, the different correlators involving $\kappa$ and $\gamma$ simplify considerably when using the Limber approximation. We now show this in more detail in a few sample derivations. First, consider $\left\langle\delta\left(\mathbf{x}_{1}\right) \kappa\left(\mathbf{x}_{2}\right)\right\rangle$, where we assume as usual $\chi_{1}<\chi_{2}$.

$$
\begin{aligned}
\left\langle\delta\left(\mathbf{x}_{1}\right) \kappa\left(\mathbf{x}_{2}\right)\right\rangle & =C \int_{0}^{\chi_{2}} d \chi \frac{W_{L}\left(\chi_{2}, \chi\right)}{a(\chi)} \xi\left(\left|\mathbf{x}_{1}-\mathbf{y}_{2}\right|\right) \\
& =C \int_{0}^{\chi_{2}} d \chi \frac{W_{L}\left(\chi_{2}, \chi\right)}{a(\chi)} \int d^{3} k e^{-i \mathbf{k} \cdot\left(\mathbf{x}_{1}-\mathbf{y}_{2}\right)} P(k) .
\end{aligned}
$$

The first expression can be used to evaluate this correlator directly. However, in the $k$-space formulation, we can apply the Limber approximation: the typical longitudinal separations $\mathbf{x}_{1 z}-\mathbf{y}_{2 z}$ are much larger than the perpendicular separation, hence the exponential will oscillate rapidly unless $k_{z} \lesssim 1 /\left(\mathbf{x}_{1 z}-\mathbf{y}_{2 z}\right)$. Thus, we set $k_{z}=0$, which yields a Dirac $\delta$-function in $\chi-\chi_{1}$, fixing the lens to be at the same distance as point 1 :

$$
\begin{aligned}
\left\langle\delta\left(\mathbf{x}_{1}\right) \kappa\left(\mathbf{x}_{2}\right)\right\rangle \stackrel{\text { L.A. }}{\simeq} C \frac{W_{L}\left(\chi_{2}, \chi_{1}\right)}{a\left(\chi_{1}\right)} \int d^{2} k_{\perp} e^{-i \mathbf{k}_{\perp} \cdot\left(\mathbf{x}_{1 \perp}-\mathbf{x}_{2 \perp}\right)} P\left(k_{\perp}\right) \\
=C \frac{W_{L}\left(\chi_{2}, \chi_{1}\right)}{a\left(\chi_{1}\right)} \widetilde{\xi}\left(x_{12 \perp}\right),
\end{aligned}
$$

where we have used the definition of the projected two-point function, equation (A8).

In a similar way, we obtain expressions for the correlators $\langle\delta \gamma\rangle$ (again assuming $\chi_{1}<\chi_{2}$ ):

$$
\left\langle\delta\left(\mathbf{x}_{1}\right) \gamma\left(\mathbf{x}_{2}\right)\right\rangle=C \int_{0}^{\chi_{2}} d \chi \frac{W_{L}\left(\chi_{2}, \chi\right)}{a(\chi)} \int d^{3} k P(k) \frac{k_{1}^{2}-k_{2}^{2}+2 i k_{1} k_{2}}{k^{2}} e^{-i \mathbf{k} \cdot\left(\mathbf{y}_{2}-\mathbf{x}_{1}\right)} .
$$

Using the Limber approximation and setting $k^{2} \rightarrow k_{\perp}^{2}$ in the denominator as well, we find

$$
\left\langle\delta\left(\mathbf{x}_{1}\right) \gamma\left(\mathbf{x}_{2}\right)\right\rangle \stackrel{\text { L.A. }}{\simeq} 2 \pi C \frac{W_{L}\left(\chi_{2}, \chi_{1}\right)}{a\left(\chi_{1}\right)} \int d^{2} k_{\perp} P\left(k_{\perp}\right) \frac{k_{1}^{2}-k_{2}^{2}+2 i k_{1} k_{2}}{k_{\perp}^{2}} e^{-i \mathbf{k}_{\perp} \cdot\left(\mathbf{x}_{2}-\mathbf{x}_{1 \perp}\right)} .
$$

The integral can be expressed as

$$
\begin{aligned}
2 \pi \int d^{2} k_{\perp} P\left(k_{\perp}\right) \frac{k_{1}^{2}-k_{2}^{2}+2 i k_{1} k_{2}}{k_{\perp}^{2}} e^{-i \mathbf{k}_{\perp} \cdot \mathbf{x}_{\perp}} & = \\
2 \pi \int d k_{\perp} k_{\perp} P\left(k_{\perp}\right) \int d \phi\left[2 \cos ^{2} \phi-1\right] e^{-i k_{\perp} x_{\perp} \cos \phi} & = \\
\frac{2 \pi}{x_{\perp}} \int d k_{\perp} P\left(k_{\perp}\right)\left[k_{\perp} x_{\perp} J_{0}\left(k_{\perp} x_{\perp}\right)-2 J_{1}\left(k_{\perp} x_{\perp}\right)\right] & =x_{\perp}^{2} \widetilde{\epsilon}\left(x_{\perp}\right),
\end{aligned}
$$

where the function $\widetilde{\epsilon}\left(x_{\perp}\right)$ is defined in equation (A13). Hence,

$$
\left\langle\delta\left(\mathbf{x}_{1}\right) \gamma\left(\mathbf{x}_{2}\right)\right\rangle \stackrel{\text { L.A. }}{\simeq} C \frac{W_{L}\left(\chi_{2}, \chi_{1}\right)}{a\left(\chi_{1}\right)} x_{12, \perp}^{2} \widetilde{\epsilon}\left(x_{12, \perp}\right) .
$$

Similarly, for the $\langle\kappa \gamma\rangle$ term we get:

$$
\left\langle\kappa\left(\mathbf{x}_{1}\right) \gamma\left(\mathbf{x}_{2}\right)\right\rangle=C^{2} \int_{0}^{\chi_{1}} d \chi \frac{W_{L}\left(\chi_{1}, \chi\right)}{a(\chi)} \int_{0}^{\chi_{2}} d \chi^{\prime} \frac{W_{L}\left(\chi_{2}, \chi^{\prime}\right)}{a\left(\chi^{\prime}\right)} \int d^{3} k P(k) \frac{k_{1}^{2}-k_{2}^{2}+2 i k_{1} k_{2}}{k^{2}} e^{-i \mathbf{k} \cdot\left(\mathbf{y}_{2}-\mathbf{y}_{1}\right)},
$$

which, applying the Limber approximation, yields

$$
\left\langle\kappa\left(\mathbf{x}_{1}\right) \gamma\left(\mathbf{x}_{2}\right)\right\rangle \stackrel{\text { L.A. }}{\simeq} C^{2} \int_{0}^{\chi_{1}} d \chi \frac{W_{L}\left(\chi_{1}, \chi\right) W_{L}\left(\chi_{2}, \chi\right)}{a^{2}(\chi)} y_{12, \perp}^{2} \widetilde{\epsilon}\left(y_{12, \perp}\right) .
$$

In all cases, the auxiliary functions are to be evaluated at $\chi_{1}$, or at $\chi$ if inside an integral over $\chi$. Fig. 11 shows the four two-point correlators as function of the transverse separation $x_{12 \perp}$, for $\mathbf{x}_{1}$ at $z=3$, and keeping $\Delta \chi_{12}=\chi_{2}-\chi_{1}$ fixed. Both the exact expressions and the Limber approximation results are shown (see the next section for a discussion). In the case of $\langle\delta \kappa\rangle,\langle\delta \gamma\rangle$, we also show the functions for different values of the longitudinal separation $\Delta \chi_{12}=\chi_{2}-\chi_{1}$. Clearly, these correlators increase strongly for increasing longitudinal separation, as they are proportional to $W_{L}\left(\chi_{2}, \chi_{1}\right) \sim \Delta \chi_{12}$. For $\langle\kappa \kappa\rangle$ and $\langle\kappa \gamma\rangle$, the dependence on the longitudinal separation of 1 and 2 is very small (as long as $\Delta \chi_{12} \ll \chi_{1}, \chi_{2}$ ), since it only enters the arguments of the lensing window functions in the integral. 


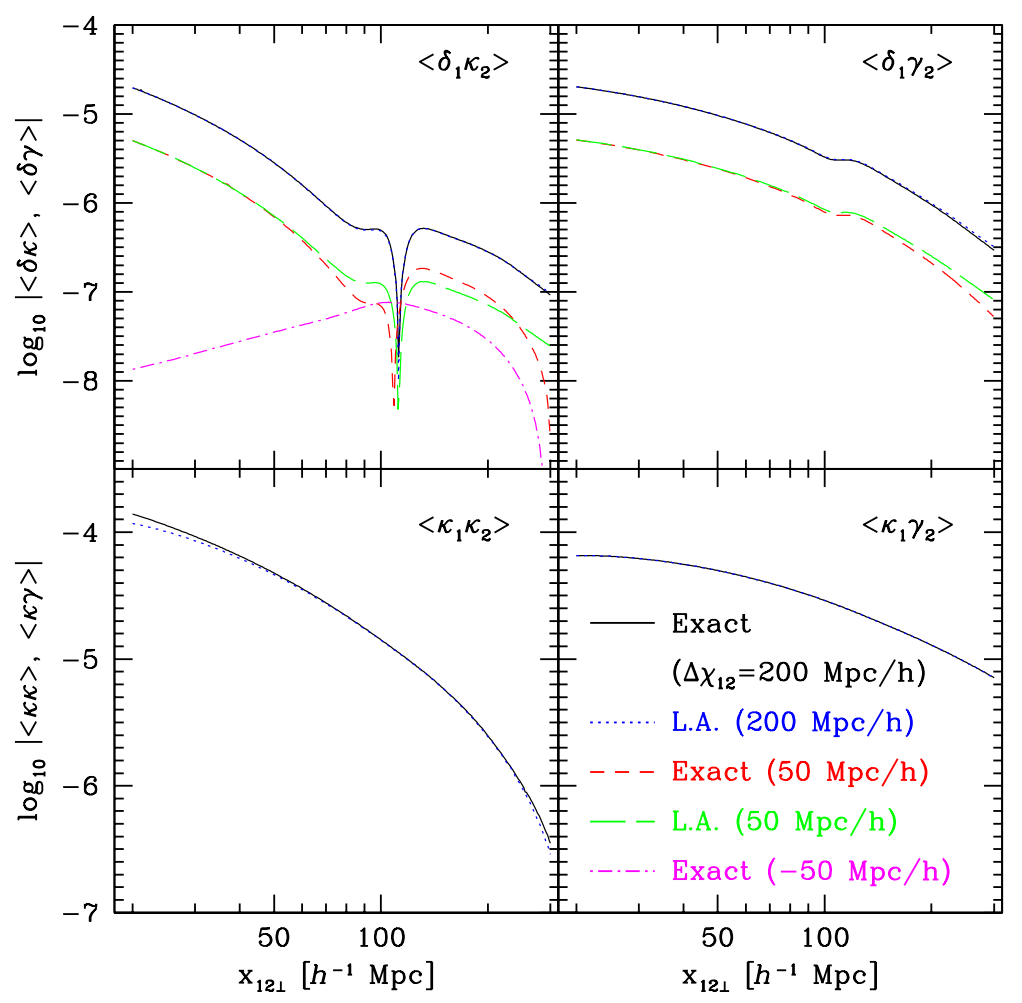

FIG. 11: $\left\langle\delta\left(\mathbf{x}_{1}\right) \kappa\left(\mathbf{x}_{2}\right)\right\rangle,\left\langle\delta\left(\mathbf{x}_{1}\right) \gamma\left(\mathbf{x}_{2}\right)\right\rangle,\left\langle\kappa\left(\mathbf{x}_{1}\right) \kappa\left(\mathbf{x}_{2}\right)\right\rangle$ and $\left\langle\kappa\left(\mathbf{x}_{1}\right) \gamma\left(\mathbf{x}_{2}\right)\right\rangle$ as function of the perpendicular separation $x_{12 \perp}$. Here, $\mathbf{x}_{1}$ is kept fixed at $z=3$, while $\mathbf{x}_{2}$ is separated by $\Delta \chi_{12}=\chi_{2}-\chi_{1}=200,50,-50 h^{-1}$ Mpc. In the last case, $\mathbf{x}_{2}$ is closer than $\mathbf{x}_{1}$. Shown are the results for the exact integral (in case of $\langle\delta \gamma\rangle$ and $\langle\kappa \gamma\rangle$, the first-order calculation) and when using the Limber approximation (L.A.).

\section{APPENDIX C: LIMBER APPROXIMATION}

\section{Discussion}

The Limber approximation is very useful to gain physical insight into the magnification effects, and for order-ofmagnitude estimates. However, before using the simplified expressions for exact calculations, one should assess the accuracy of this approximation. We calculated the exact integrals for all of the terms in equations (37)-(43), except the $\langle\kappa \kappa \kappa\rangle,\langle\delta \gamma\rangle$, and $\langle\kappa \gamma\rangle$ terms. For the latter two terms involving the shear, the exact calculation is very difficult, and we include the first order correction which is good to $O\left(k_{z}^{4} / k_{\perp}^{4}\right)$ instead. The calculation of this correction is outlined in the next section. Note that the first order correction to the shear terms are quite small, so that there is no need to go to higher orders.

Fig. 11 shows a comparison of the exact expressions and the Limber approximation. For $\langle\delta \kappa\rangle$ and $\langle\delta \gamma\rangle$, the Limber approximation is not accurate anymore at large transverse separations. Since we keep the longitudinal separation $\Delta \chi_{12}$ fixed, large $x_{12 \perp}$ correspond to more and more transverse $\mathbf{x}_{12}$. Correspondingly, the deviations become smaller for larger $\Delta \chi_{12}$, as is apparent from Fig. 11. The reason for this is that the Limber approximation assumes that separation vectors with $x_{\|} \lesssim x_{\perp}$ contribute to the correlation. However, if $x_{12 \perp}>x_{12 \|}$, then this overcounts the contributing modes and overestimates $\langle\delta \kappa\rangle,\langle\delta \gamma\rangle$. As we lower $x_{12 \|}=\Delta \chi_{12}$ to 0 and negative values, the result of the Limber approximation goes linearly to 0 , while there is actually still a finite correlation at negative $\Delta \chi_{12}$, i.e. when we correlate an overdensity at $\mathbf{x}_{1}$ behind the lens magnifying $\mathbf{x}_{2}$. Assuming the lens is a distance $\Delta \chi_{L}$ in front of $\mathbf{x}_{2}$, an order of magnitude estimate gives $\left\langle\delta_{1} \kappa_{2}\right\rangle \sim\left(H_{0} \Delta \chi_{L}\right)\left(H_{0} x_{12 \perp}\right) \xi\left(r_{L}\right)$, where $r_{L}=\sqrt{x_{12 \perp}^{2}+\left(\Delta \chi_{L}+\left|\Delta \chi_{12}\right|\right)^{2}}$. For $x_{12 \perp}=100 h^{-1} \mathrm{Mpc}, \Delta \chi_{12}=-50 h^{-1} \mathrm{Mpc}$, and setting $\Delta \chi_{L} \sim 50 h^{-1} \mathrm{Mpc}$, this yields $\left|\left\langle\delta_{1} \kappa_{2}\right\rangle\right| \sim 10^{-8}$. This is in rough agreement with the exact calculation, shown by the dash-dotted curve of $\left\langle\delta_{1} \kappa_{2}\right\rangle$ for $\Delta \chi_{12}=-50$ in Fig. 11 . Clearly, $\left\langle\delta_{1} \kappa_{2}\right\rangle$ becomes very small for negative $\Delta \chi_{12}$ and in fact decreases rapidly for increasingly negative values. Terms like this would only contribute for almost transverse separations, where the $\langle\delta \kappa\rangle$ term is in any case very small. 
This justifies the neglection of terms involving $\left\langle\delta_{2} \kappa_{1}\right\rangle$ etc. in the derivation of equations (18)-(31).

For the $\langle\kappa \kappa\rangle$ and $\langle\kappa \gamma\rangle$ terms, the Limber approximation is generally very good (we do not show them for other values of $\Delta \chi_{12}$ as they are essentially the same). For $\langle\kappa \kappa\rangle$, there are very small deviations at small $x_{12 \perp}$, corresponding to almost longitudinal $\mathbf{x}$. In this case, the Limber approximation underestimates the correlation, counting only purely transverse modes $\left(y_{12 \|}<y_{12 \perp}\right)$, while in the exact calculation non-transverse modes contribute as well.

To summarize, we found that the Limber approximation holds well for quantities that, after the approximation, still involve an integral over the line of sight, like $\langle\kappa \kappa\rangle,\langle\kappa \gamma\rangle$, and $\langle\kappa \kappa \kappa\rangle$. The integral serves to average out the effect of the approximation. For terms that only involve a single evaluation of the correlation functions, e.g., $\langle\delta \kappa\rangle,\langle\delta \gamma\rangle$, $\langle\delta \kappa \kappa\rangle$, the Limber approximation shows deviations, especially when the Limber-approximated expression goes to zero, while the actual correlation is finite. For these quantities, the exact integral should be used.

\section{Shear terms beyond the Limber approximation}

While the evaluation of the exact integrals is straightforward for the terms involving the convergence $\kappa$, going beyond the Limber approximation requires more work for the shear terms - however, it provides some insight as well. Going back to equation (B11) and using cylindrical coordinates $\left(k_{\perp}, k_{z}, \phi\right)$, we can write $\left\langle\delta_{1} \gamma_{2}\right\rangle$ as:

$$
\begin{aligned}
\left\langle\delta\left(\mathbf{x}_{1}\right) \gamma\left(\mathbf{x}_{2}\right)\right\rangle & =C \int_{0}^{\chi_{2}} d \chi \frac{W_{L}\left(\chi_{2}, \chi\right)}{a(\chi)} I\left(\mathbf{r}=\mathbf{y}_{2}-\mathbf{x}_{1}, \chi\right) \\
I(\mathbf{r}, \chi) & =\int k_{\perp} d k_{\perp} \int d k_{z} P\left(k=\sqrt{k_{\perp}^{2}+k_{z}^{2}} ; \chi\right) \int d \phi \frac{k_{\perp 1}^{2}-k_{\perp 2}^{2}+2 i k_{\perp 1} k_{\perp 2}}{k^{2}} \exp \left(-i\left(\mathbf{k}_{\perp} \cdot \mathbf{r}_{\perp}+k_{z} r_{\|}\right)\right) \\
& =\int k_{\perp} d k_{\perp} \int d k_{z} \exp \left(-i k_{z} r_{\|}\right) P(k ; \chi) \frac{k_{\perp}^{2}}{k^{2}} \int d \phi\left(2 \cos ^{2} \phi-1+2 i \cos \phi \sin \phi\right) \exp \left(-i\left(\mathbf{k}_{\perp} \cdot \mathbf{r}_{\perp}\right)\right) \\
& =\int k_{\perp} d k_{\perp} \int d k_{z} \exp \left(-i k_{z} r_{\|}\right) P(k ; \chi) \frac{k_{\perp}^{2}}{k_{\perp}^{2}+k_{z}^{2}}(2 \pi)\left(J_{0}\left(k_{\perp} r_{\perp}\right)-\frac{2}{k_{\perp} r_{\perp}} J_{1}\left(k_{\perp} r_{\perp}\right)\right)
\end{aligned}
$$

where we have aligned $\mathbf{k}_{\perp}$ so that $\phi$ is the angle between $\mathbf{k}_{\perp}$ and $\mathbf{r}_{\perp}$. The last integral is difficult to evaluate. In order to make progress, we use the fact that typically $r_{\|} \gg r_{\perp}$, so that $k_{z}$ should be much less than $k_{\perp}$ to get a significant contribution. Thus, we expand the integrand in powers of $k_{z}^{2} / k_{\perp}^{2}$. For the power spectrum, this expansion yields:

$$
P(k)=P\left(k_{\perp}\right)+\left.\frac{1}{2 k_{\perp}} \frac{\partial P}{\partial k}\right|_{k_{z}=0} k_{z}^{2}+O\left(\frac{k_{z}}{k_{\perp}}\right)^{4} .
$$

Here and in the following, we have suppressed the $\chi$-evolution of the power spectrum. Note that in this expansion there is no linear (and in general no odd) term in $k_{z}$. We now obtain

$$
\begin{aligned}
I(\mathbf{r}) \simeq & \int k_{\perp} d k_{\perp} \int d k_{z} \exp \left(-i k_{z} r_{\|}\right)\left(P\left(k_{\perp}\right)+\left.\frac{1}{2 k_{\perp}} \frac{\partial P}{\partial k}\right|_{k_{z}=0} k_{z}^{2}\right)\left(1-\frac{k_{z}^{2}}{k_{\perp}^{2}}\right) \\
& \times(2 \pi)\left(J_{0}\left(k_{\perp} r_{\perp}\right)-\frac{2}{k_{\perp} r_{\perp}} J_{1}\left(k_{\perp} r_{\perp}\right)\right) .
\end{aligned}
$$

We then obtain zeroth and first order expressions for the integral, the first order expression being good to $\left(k_{z} / k_{\perp}\right)^{4}$ :

$$
\begin{aligned}
I^{(0)}(\mathbf{r})= & \int k_{\perp} d k_{\perp} P\left(k_{\perp}\right)(2 \pi)\left(J_{0}\left(k_{\perp} r_{\perp}\right)-\frac{2}{k_{\perp} r_{\perp}} J_{1}\left(k_{\perp} r_{\perp}\right)\right) \int d k_{z} \exp \left(-i k_{z} r_{\|}\right) \\
I^{(1)}(\mathbf{r})= & \int k_{\perp} d k_{\perp}\left(-\frac{P\left(k_{\perp}\right)}{k_{\perp}^{2}}+\left.\frac{1}{2 k_{\perp}} \frac{\partial P}{\partial k}\right|_{k_{z}=0}\right)(2 \pi)\left(J_{0}\left(k_{\perp} r_{\perp}\right)-\frac{2}{k_{\perp} r_{\perp}} J_{1}\left(k_{\perp} r_{\perp}\right)\right) \\
& \times \int d k_{z} \exp \left(-i k_{z} r_{\|}\right) k_{z}^{2} .
\end{aligned}
$$

The integrals over $k_{z}$ result in Dirac $\delta_{D}$ functions and derivatives. We see that the zeroth order term is exactly what we obtained earlier in the Limber approximation, equation (B14):

$$
I^{(0)}(\mathbf{r})=\frac{(2 \pi)^{2}}{r_{\perp}} \int d k P(k)\left(k r_{\perp} J_{0}\left(k r_{\perp}\right)-2 J_{1}\left(k r_{\perp}\right)\right)=r_{\perp}^{2} \widetilde{\epsilon}\left(r_{\perp}\right) \delta_{D}\left(r_{\|}\right)
$$


where $r_{\|}=\chi-\chi_{1}$ in equation $(\mathrm{C} 1)$, so that the $\delta_{D}$ function fixes $\chi=\chi_{1}$. For the first correction to the Limber approximation, we get

$$
I^{(1)}(\mathbf{r})=\frac{(2 \pi)^{2}}{r_{\perp}} \int \frac{d k}{k^{2}} P(k)\left(\frac{1}{2} \frac{\partial \ln P}{\partial \ln k}-1\right)\left(k r_{\perp} J_{0}\left(k r_{\perp}\right)-2 J_{1}\left(k r_{\perp}\right)\right) \cdot \frac{\partial^{2}}{\partial r_{\|}^{2}} \delta_{D}\left(r_{\|}\right) \equiv \widetilde{\nu}\left(r_{\perp}\right) \cdot \frac{\partial^{2}}{\partial r_{\|}^{2}} \delta_{D}\left(r_{\|}\right),
$$

defining another auxiliary function $\widetilde{\nu}(r)$. By partial integration in equation $(\mathrm{C} 1)$ for $\langle\delta \gamma\rangle$ and equation $(\mathrm{B} 15)$ for $\langle\kappa \gamma\rangle$, we can calculate the first correction to the Limber approximation for the shear terms, $\langle\delta \gamma\rangle^{(1)},\langle\kappa \gamma\rangle^{(1)}$ :

$$
\begin{aligned}
& \langle\delta \gamma\rangle^{(1)}=-C \frac{\partial^{2}}{\partial \chi_{1}^{2}}\left(\frac{W_{L}\left(\chi_{2}, \chi_{1}\right)}{a\left(\chi_{1}\right)}\right) \widetilde{\nu}\left(x_{12 \perp} ; \chi_{1}\right) \\
& \langle\kappa \gamma\rangle^{(1)}=-C^{2} \int_{0}^{\chi_{1}} d \chi \frac{\partial^{2}}{\partial \chi^{2}}\left(\frac{W_{L}\left(\chi_{2}, \chi\right) W_{L}\left(\chi_{1}, \chi\right)}{a^{2}(\chi)}\right) \widetilde{\nu}\left(y_{12 \perp} ; \chi\right),
\end{aligned}
$$

where $y_{12 \perp}=\left(\chi / \chi_{1}\right) x_{12 \perp}$ and we have made the dependence on the evolution $\chi$ explicit again. The derivatives of the lensing weight factors can be done easily, yielding polynomials in $\chi_{1}, \chi$ and factors of $H(z)$ and $d H / d z$.

The effect of the first order correction on $\left\langle\delta_{1} \gamma_{2}\right\rangle$ and $\left\langle\kappa_{1} \gamma_{2}\right\rangle$ is shown in Fig. 11 (right two plots). While for $\left\langle\delta_{1} \gamma_{2}\right\rangle$ there is some effect at large $x_{12} \perp$ (similarly to $\left.\left\langle\delta_{1} \kappa_{2}\right\rangle\right)$, the correction to $\left\langle\kappa_{1} \gamma_{2}\right\rangle$ is negligible. In any case, we conclude that the first-order calculation is sufficient, and there is no need to go to higher orders in equation (C4).

\section{APPENDIX D: A ROUGH ERROR ESTIMATE FOR THE THREE-POINT CORRELATION FUNCTION}

In order to estimate the error on the three-point correlation function (3PCF), we start from the number of triples $D D D$ observed, i.e. the number of triangles in a certain geometry bin formed by galaxies in a survey. Say the survey observes a total volume $V$ containing $N_{g}$ galaxies. We specify a triangle bin in such a way that, after fixing point 1 , we count all triangles where a galaxy is found in the volume element $d V_{2}$ around point 2 and a galaxy found in $d V_{3}$ around point 3 (points 2 and 3 being determined by the triangle geometry considered). Then, the expectation value of triples is given by:

$$
\begin{aligned}
\langle D D D\rangle & =N_{3}\left[1+\xi\left(r_{12}\right)+\xi\left(r_{13}\right)+\xi\left(r_{23}\right)+\zeta\left(r_{12}, r_{13}, r_{23}\right)\right] \\
N_{3} & \equiv N_{g}\left(n_{g} d V_{2}\right)\left(n_{g} d V_{3}\right)=N_{g}^{3} \frac{d V_{2}}{V} \frac{d V_{3}}{V}
\end{aligned}
$$

where $n_{g}$ is the average volume density of galaxies, estimated by $n_{g}=N_{g} / V$. An estimator for the $3 \mathrm{PCF} \zeta$ is given in $[59], \S 55$ :

$$
\hat{\zeta}=\frac{D D D-D D R}{R R R}+2
$$

where $D$ stands for points drawn from the data, while $R$ stands for points drawn from a random distribution (with the same boundaries). Calculalting the variance of this estimator, we obtain:

$$
\begin{aligned}
\sigma^{2}(\hat{\zeta}) \equiv & \left\langle\hat{\zeta}^{2}\right\rangle-\langle\hat{\zeta}\rangle^{2} \\
= & \left\langle\frac{(D D D)^{2}}{(R R R)^{2}}\right\rangle-\left\langle\frac{D D D}{R R R}\right\rangle^{2}-2\left\langle\frac{D D D D D R}{(R R R)^{2}}\right\rangle \\
& +2\left\langle\frac{D D D}{R R R}\right\rangle\left\langle\frac{D D R}{R R R}\right\rangle+\left\langle\frac{(D D R)^{2}}{(R R R)^{2}}\right\rangle-\left\langle\frac{D D R}{R R R}\right\rangle^{2} .
\end{aligned}
$$

To proceed further, we make the following approximations. The $R R R$ in the denominator is assumed to be uncorrelated with the numerators. Further, we set:

$$
\left\langle(R R R)^{2}\right\rangle=\langle R R R\rangle^{2}=N_{3}^{2}
$$

In addition, if we assume that the galaxy correlations are weak, i.e. that $\langle D D D\rangle \approx N_{3}$, then the second and third set of terms in equation (D5) vanish. We then obtain:

$$
\begin{aligned}
\sigma^{2}(\hat{\zeta}) & =\frac{1}{N_{3}^{2}}\left(\left\langle(D D D)^{2}\right\rangle-\langle D D D\rangle^{2}\right) \\
& =\frac{\sigma^{2}(\langle D D D\rangle)}{N_{3}^{2}}
\end{aligned}
$$


In this approximation, the error on the $3 \mathrm{PCF} \zeta$ is simply the relative error on the number of triples $D D D$. The latter was calculated in [60] and is given to good accuracy by:

$$
\sigma^{2}(\langle D D D\rangle)=\langle D D D\rangle+\frac{36}{N_{g}}\langle D D D\rangle^{2},
$$

The first term is the standard Poisson term expected for any number count. The second one was found for the first time in the derivation of [60]. Now we again neglect the galaxy correlations in equation (D1) and set $\langle D D D\rangle \approx N_{3}=$ $N_{g} n_{g}^{2} d V_{2} d V_{3}$, to obtain:

$$
\sigma(\zeta)=\sqrt{\frac{1}{\langle D D D\rangle}+\frac{36}{N_{g}}}=N_{g}^{-1 / 2} \sqrt{\frac{1}{n_{g}^{2} d V_{2} d V_{3}}+36} .
$$

The first term again is the Poisson term: $\sigma(\zeta)_{\text {Poisson }}=N_{\text {triples }}^{-1 / 2}$, analogous to $\sigma(\xi) \approx N_{\text {pairs }}^{-1 / 2}$ as found in [59], $\S 48$, for the two-point correlation function. The second term in equation (D8) was added in [60], along with a similar term for the variance of pair counts. 